\title{
A Simplified Milstein Scheme for SPDEs with Multiplicative Noise
}

\author{
B. Ghayebi and S. M. Hosseini \\ Department of Applied Mathematics, Tarbiat Modares University, P.O. Box 14115-175, Tehran, Iran \\ Correspondence should be addressed to B. Ghayebi; b.ghayebi@modares.ac.ir
}

Received 3 May 2014; Revised 21 June 2014; Accepted 29 June 2014; Published 4 August 2014

Academic Editor: Reza Ezzati

Copyright (C) 2014 B. Ghayebi and S. M. Hosseini. This is an open access article distributed under the Creative Commons Attribution License, which permits unrestricted use, distribution, and reproduction in any medium, provided the original work is properly cited.

\begin{abstract}
This paper deals with a research question raised by Jentzen and Röckner (A Milstein scheme for SPDEs, arXiv:1001.2751v4 (2012)), whether the exponential term in their introduced scheme can be replaced by a simpler mollifier. This replacement can lead to more simplification and computational reduction in simulation. So, in this paper, we essentially replace the exponential term with a Padé approximation of order 1 and denote the resulting scheme by simplified Milstein scheme. The convergence analysis for this scheme is carried out and it is shown that even with this replacement the order of convergence is maintained, while the resulting scheme is easier to implement and slightly more efficient computationally. Some numerical tests are given that confirm the order of accuracy and also computational cost reduction.
\end{abstract}

\section{Introduction}

Many models in engineering, physics, complex phenomena, and so forth are described by stochastic partial differential equations (SPDEs); for example, see [1-6]. Since the exact solutions of these equations are rarely known, the numerical analysis of SPDEs has been recently the subject of many papers; for example, see [7-14], for more detailed discussion on this topic and many examples in applied sciences. In this paper, we consider strong approximation (see [4, Section 9.3]) of SPDEs of evolutionary type. To demonstrate the results of this paper clearly, we focus on the following example of SPDE:

$$
\begin{aligned}
d X_{t}(x)= & {\left[\lambda \frac{\partial^{2}}{\partial x^{2}} X_{t}(x)+f\left(x, X_{t}(x)\right)\right] d t } \\
& +b\left(x, X_{t}(x)\right) d W_{t}(x),
\end{aligned}
$$

with initial condition $X_{0}(x)=\xi(x)$ and Dirichlet boundary conditions $X_{t}(0)=X_{t}(1)=0$ for all $x \in(0,1)$ and $t \in[0, T]$, where $\lambda \in(0, \infty)$. Let $(\Omega, F, P)$ be a probability space with a normal filtration $\left(\mathscr{F}_{t}\right)_{t \in[0, T]}$, let $H=$ $L^{2}((0,1), \mathbb{R})$ be the $\mathbb{R}$-Hilbert space of equivalence classes of Lebesgue square integrable functions from $(0,1)$ to $\mathbb{R}$, and let $f, b:(0,1) \times \mathbb{R} \rightarrow \mathbb{R}$ be two appropriate smooth and regular functions with globally bounded derivatives. Let $W$ : $[0, T] \times \Omega \rightarrow H$ be a standard $Q$-Wiener process with regard to $\left(\mathscr{F}_{t}\right)_{t \in[0, T]}$, with a trace class operator $Q: H \rightarrow H$ and $\xi:[0,1] \rightarrow \mathbb{R}$ with $\xi(0)=\xi(1)=0$ being a smooth function. The covariance operator $Q: H \rightarrow H$ has orthonormal basis $g_{j} \in H, j \in \mathbb{N}$, of eigenfunctions with summable eigenvalues $\mu_{j} \in[0, \infty) j \in \mathbb{N}$. Under the previous assumption, the SPDE (1) has a unique mild solution. Specifically, there exists an up to unpredictable unique adapted stochastic process $X$ : $[0, T] \times \Omega \rightarrow H$ with continuous sample path which satisfies

$$
\begin{aligned}
X_{t}= & e^{A t} \xi+\int_{0}^{t} e^{A(t-s)} F\left(X_{s}\right) d s \\
& +\int_{0}^{t} e^{A(t-s)} B\left(X_{s}\right) d W_{s},
\end{aligned}
$$

for all $t \in[0, T]$, where $A: D(A) \subset H \rightarrow H$ is the Laplacian with Dirichlet boundary conditions times the constant $\lambda \epsilon$ $(0, \infty)$ and where $F: H \rightarrow H$ and $B: H \rightarrow H S(U, H)$ are given by $(F(v))(x)=f(x, v(x))$ and $(B(v) u)(x)=b(x, v(x))$. $u(x)$ for all $x \in(0,1), v \in H$ and all $u \in U_{0}$ where $U_{0}=Q^{1 / 2}$ with $\langle v, w\rangle_{U_{0}}=\left\langle Q^{-1 / 2} v, Q^{-1 / 2} w\right\rangle$ for all $v, w \in U_{0}$ is the image 
$\mathbb{R}$-Hilbert space of $Q^{1 / 2}$ (see [15, Appendix C]); note that $A$ and $Q$ commutate in our example SPDE (2). Now we are concerned about the strong approximation of the SPDE (1). More formally we want to compute numerical approximation $Y: \Omega \rightarrow H$ such that

$$
\begin{aligned}
& \left(E\left\|X_{T}-Y\right\|_{H}^{2}\right)^{1 / 2} \\
& \quad:=\left(E\left[\int_{(0,1)}\left|X_{T}(x)-Y(x)\right|^{2} d x\right]\right)^{1 / 2}<\varepsilon
\end{aligned}
$$

holds for a given precision $\varepsilon>0$ with the least possible computational effort. To simulate the numerical approximation on a computer, one has to discretize both the time interval $[0, T]$ and the infinite dimensional space $H=L^{2}((0,1), \mathbb{R})$. In this paper we consider spectral Galerkin for spatial discretization and difference method for temporal discretization. A simple full discretization for (1) is the linear implicit Euler method combined with spectral Galerkin method which is given by

$$
\begin{aligned}
\bar{Y}_{k+1}^{N}= & P_{N}\left(I-\frac{T}{N^{3}} A\right)^{-1} \\
& \times\left(\bar{Y}_{k}^{N}+\frac{T}{N^{3}} f\left(\cdot, \bar{Y}_{k}^{N}\right)\right. \\
& \left.\quad+b\left(\cdot, \bar{Y}_{k}^{N}\right)\left(W_{(k+1) T / N^{3}}^{N}-W_{k T / N^{3}}^{N}\right)\right),
\end{aligned}
$$

$k=0,1, \ldots, N^{3}-1$, and all $N \in \mathbb{N}$, with $\bar{Y}_{0}^{N}=\xi^{N}$ and $\xi^{N}=$ $P_{N}(\xi)$, where $P_{N}$ is a bounded linear operator such that $P_{N}$ : $H \rightarrow H$ with

$$
\left(P_{N}(v)\right)(x)=\sum_{n=1}^{N} 2 \sin (n \pi x) \int_{0}^{1} \sin (n \pi y) v(y) d y
$$

for all $x \in(0,1), v \in H$, and $N \in \mathbb{N}$, and the finite dimensional Wiener processes $W^{N}:[0, T] \times \Omega \rightarrow H, N \in \mathbb{N}$, are given by $W_{t}^{N}(\omega)=P_{N}\left(W_{t}(\omega)\right)$ for all $t \in[0, T], \omega \in \Omega$, and $N \in \mathbb{N}$. According to the analysis of [16], for method (4) with $k=N^{3}$, there exist real numbers $C_{r}>0$ that for small $r \in(0,3 / 2)$

$$
\left(E\left\|X_{T}-Y_{N^{3}}^{N}\right\|_{H}^{2}\right)^{1 / 2} \leq C_{r} N^{r-3 / 2}
$$

holds for all $N \in \mathbb{N}$. This means that the method has overall convergence $3 / 8$ - (for a real number $\beta \in(0, \infty)$, we write $\beta$ for the convergence order if the convergence order is higher in order than $\beta-\epsilon$ for all arbitrary small $\epsilon \in(0, \beta))$. In [16] Jentzen and Rockner proposed an infinite dimensional analog of Milstein type scheme for (1) given by $\widetilde{Y}_{0}^{N}=\xi_{0}^{N}=$ $P_{N}(\xi)$ and

$$
\begin{aligned}
\tilde{Y}_{k+1}^{N}= & P_{N} e^{A\left(T / N^{2}\right)} \\
& \times\left(\tilde{Y}_{k}^{N}+\frac{T}{N^{2}} f\left(\cdot, \widetilde{Y}_{k}^{N}\right)\right. \\
& +b\left(\cdot, \widetilde{Y}_{k}^{N}\right)\left(W_{(k+1) T / N^{2}}^{N}-W_{k T / N^{2}}^{N}\right) \\
& +\frac{1}{2}\left(\frac{\partial}{\partial y} b\right)\left(\cdot, \tilde{Y}_{k}^{N}\right) b\left(\cdot, \tilde{Y}_{k}^{N}\right) \\
& \left.\times\left(\left(W_{(k+1) T / N^{2}}^{N}-W_{k T / N^{2}}^{N}\right)^{2}-\frac{T}{N^{2}} \sum_{i=1}^{N} \eta_{i} g_{i}^{2}\right)\right)
\end{aligned}
$$

for all $k \in\left\{0,1, \ldots, N^{2}-1\right\}$ and $N \in \mathbb{N}$. Here we use the notations $v \cdot w:(0,1) \rightarrow \mathbb{R}, v^{2}:(0,1) \rightarrow \mathbb{R}$, and $(\phi(\cdot, v))(x)=\phi(x, v(x))$ for all $x \in(0,1)$ and all functions $v, w:(0,1) \rightarrow \mathbb{R}, \phi:(0,1) \times \mathbb{R} \rightarrow \mathbb{R}$. Method (7) gives a break of complexity of the numerical approximation of nonlinear SPDE with multiplicative trace class noise. More precisely, it is shown in [16] that $N^{2}$ time steps in contrast to $N^{3}$ time steps for the linear implicit Euler scheme (4) are required to achieve (6). That is the Milstein type scheme (7) with $N^{2}$ time steps guarantees that for real numbers $C_{r}>0$, $r \in(0,3 / 2)$, such that

$$
\left(E\left\|X_{T}-Y_{N^{2}}^{N}\right\|_{H}^{2}\right)^{1 / 2} \leq C_{r} N^{r-3 / 2}
$$

holds for all $N \in \mathbb{N}$. Thus the scheme has the overall convergence order of $1 / 2-$. Consequently scheme (7) increases the overall convergence order from $3 / 8-$ to $1 / 2-$. As mentioned before, in this paper essentially the exponential term in the Milstein type scheme [16] is replaced by a first order approximation which makes the scheme easier to implement and slightly more efficient computationally while preserving the order of convergence. The analysis and implementation will be carried out as follows. In Section 2 the required setting and assumptions are formulated. In Section 3 the simplified Milstein scheme is formulated. In Section 4 we state and prove the main result of this section concerning the convergence of the simplified Milstein scheme. Finally in Section 5 numerical example for a stochastic reaction diffusion equation is presented to show numerically the order of convergence and computational costs. The numerical simulations will be carried out in MATLAB environment on a PC with CPU $2.66 \mathrm{GHz}$.

\section{Setting and Assumptions}

Throughout this paper suppose that the setting and following assumptions are fulfilled. Fix $T \in(0, \infty)$. Let $(\Omega, F, P)$ be a probability space with a normal filtration $\left\{F_{t}\right\}_{t \in[0, T]}$ and let $\left(H,\langle\rangle,,\|\cdot\|_{H}\right)$ and $\left(U,\langle\rangle,,\|\cdot\|_{U}\right)$ be two separable $\mathbb{R}$-Hilbert spaces. Moreover, let $W:[0, T] \times \Omega \rightarrow U$ be a standard $Q$ Wiener process with respect to $\left\{F_{t}\right\}_{t \in[0, T]}$, with a trace class operator $Q: U \rightarrow U$. 
Assumption 1 (linear operator $A$ ). Let $A: D(A) \subset H \rightarrow H$ be a linear operator such that

$$
A v=-\sum_{i \in \mathbb{N}} \lambda_{i}\left\langle e_{i}, v\right\rangle_{H} e_{i}
$$

for every $v \in D(A)$ with $D(A)=\left\{\left.w \in H\left|\sum_{i \in \mathbb{N}}\right| \lambda_{i}\right|^{2}\right.$ $\left.\left|\left\langle e_{i}, w\right\rangle_{H}\right|^{2}<\infty\right\}$.

Here $\left(\lambda_{i}\right)_{i \in \mathbb{N}}$ is a family of real numbers with $\inf _{i \in \mathbb{N}} \lambda_{i} \in$ $(0, \infty)$ and $\left(e_{i}\right)_{i \in \mathbb{N}}$ is an orthonormal basis of $H$. By $V_{r}:=$ $D\left((-A)^{r}\right)$ equipped with the norm $\|v\|_{V_{r}}=\left\|(-A)^{r} v\right\|_{H}$ for all $v \in V_{r}, 0 \leq r$, we denote the $\mathbb{R}$-Hilbert space of domains of fractional powers of the linear operator $-A: D(A) \rightarrow H$.

Assumption 2 (drift term $F$ ). Let $\beta \in[0,1)$ be a real number and let $F: V_{\beta} \rightarrow H$ be a globally Lipschitz continuous; that is, $\sup _{v, w \in H}\left(\|F(v)-F(w)\|_{H} /\|v-w\|_{H}\right)<\infty$ and $\|F(v)\|_{H}<$ $c\left(1+\|v\|_{V_{\beta}}\right), c \in(0, \infty)$; in addition

$$
\begin{gathered}
\sup _{v \in V_{\beta}}\left\|F^{\prime}(v)\right\|_{L(H)}<\infty, \\
\sup _{v \in V_{\beta}}\left\|F^{\prime \prime}(v)\right\|_{L^{(2)}\left(V_{\beta}, H\right)}<\infty .
\end{gathered}
$$

Assumption 3 (diffusion term $B$ ). Let $B: V_{\beta} \rightarrow H S\left(U_{0}\right.$, $H)$ be a globally Lipschitz continuous mapping and twice continuously Frechet differentiable mapping with $\sup _{v \in V_{\beta}}\left\|B^{\prime}(v)\right\|_{L\left(H, H S\left(U_{0}, H\right)\right)}<\infty$ and $\sup _{v \in V_{\beta}}\left\|B^{\prime \prime}(v)\right\|_{L^{(2)}\left(V_{\beta}, H S\left(U_{0}, H\right)\right)}<\infty$. In addition $\alpha \in(0, \infty)$, $\delta, \theta \in(0,1 / 2)$ with $\beta \leq \delta+1 / 2, \gamma \in[\max (\delta, \beta), \delta+1 / 2)$, and $c \in(0, \infty)$ is a real number such that $B\left(V_{\delta}\right) \subset H S\left(U_{0}, V_{\delta}\right)$ and

$$
\begin{gathered}
\|B(u)\|_{H S\left(U_{0}, V_{\delta}\right)} \leq c\left(1+\|u\|_{V_{\delta}}\right), \\
\left\|B^{\prime}(v) B(v)-B^{\prime}(w) B(w)\right\|_{H S^{(2)}\left(U_{0}, H\right)} \leq c\|v-w\|_{H}, \\
\left\|(-A)^{-9} B(v) Q^{-\alpha}\right\|_{H S\left(U_{0}, H\right)} \leq c\left(1+\|v\|_{V_{\gamma}}\right),
\end{gathered}
$$

hold for all $u \in V_{\delta}$ and $v, w \in V_{\gamma}$. Additionally, let the bilinear Hilbert-Schmidt operator $B^{\prime}(v) B(v) \in H S^{(2)}\left(U_{0}, H\right)$ be symmetric for all $v \in V_{\beta}$. Note that the operator $B^{\prime}(v) B(v)$ : $U_{0} \times U_{0} \rightarrow H$, given by

$$
\left(B^{\prime}(v) B(v)\right)(u, \widetilde{u})=\left(B^{\prime}(v)(B(v) u)\right) \tilde{u}
$$

for all $u, \tilde{u} \in U_{0}$, is a bilinear Hilbert-Schmidt operator in $H S^{(2)}\left(U_{0}, H\right)$ for all $v \in V_{\beta}$.

The assumed symmetry of $B^{\prime}(v) B(v) \in H S^{(2)}\left(U_{0}, H\right)$ thus reads as [16, Remark 1$]$.

Assumption 4 (initial value $\xi$ ). Let $\xi: \Omega \rightarrow V_{\gamma}$ be an $F_{0} / B\left(V_{\gamma}\right)$-measurable mapping with $E\|\xi\|_{V_{\gamma}}^{2}<\infty$.
Proposition 5 (existence of the mild solution). Let $T>0$. Then under Assumptions 1-4, there exists an up to modifications unique predictable stochastic process $X:[0, T] \times \Omega \rightarrow V_{\gamma}$ which fulfills $\sup _{t \in[0, T]} E\left\|X_{t}\right\|_{V_{\gamma}}^{2}<\infty$,

$$
\begin{gathered}
\sup _{t \in[0, T]} E\left\|B\left(X_{t}\right)\right\|_{H S\left(U_{0}, V_{\delta}\right)}^{2}<\infty, \\
X_{t}=e^{A t} \xi+\int_{0}^{t} e^{A(t-s)} F\left(X_{s}\right) d s \\
+\int_{0}^{t} e^{A(t-s)} B\left(X_{s}\right) d W_{s},
\end{gathered}
$$

for all $t \in[0, T]$; moreover, we have

$$
\sup _{t_{1}, t_{2} \in[0, t]} \frac{\left(E\left\|X_{t_{2}}-X_{t_{1}}\right\|_{H}^{2}\right)^{1 / 2}}{\left|t_{2}-t_{1}\right|^{\min (\gamma, 1 / 2)}}<\infty .
$$
[17].

Proposition 5 immediately follows from Theorem 1 in

\section{The Proposed Simplified Milstein Scheme}

We construct the simplified Milstein scheme for nonlinear stochastic partial differential equations. For this work first we use Taylor formula in Banach space for coefficients $B$ and $F$ for the problem (2). More formally using $F\left(X_{s}\right) \approx F\left(X_{0}\right)$ and $B\left(X_{s}\right) \approx B\left(X_{0}\right)+B^{\prime}\left(X_{0}\right)\left(X_{s}-X_{0}\right)$ for small $s \in[0, T]$ shows

$$
\begin{aligned}
X_{t}= & e^{A t} \xi+\int_{0}^{t} e^{A(t-s)} F\left(X_{0}\right) d s \\
& +\int_{0}^{t} e^{A(t-s)} B^{\prime}\left(X_{0}\right)\left(X_{s}-X_{0}\right) d W_{s}
\end{aligned}
$$

for small $t \in[0, T]$. Using the approximation $X_{s} \approx X_{0}+$ $\int_{0}^{s} B\left(X_{0}\right) d W_{u}$ for small $s \in[0, T]$ gives

$$
\begin{aligned}
X_{t} \approx & e^{A t} X_{0}+t e^{A t} F\left(X_{0}\right) \\
& +\int_{0}^{t} e^{A t} B\left(X_{0}\right) d W_{s} \\
& +\int_{0}^{t} e^{A t} B^{\prime}\left(X_{0}\right)\left(\int_{0}^{s} B\left(X_{0}\right) d W_{u}\right) d W_{s} .
\end{aligned}
$$

We then substitute $e^{A t} \approx(I-t A)^{-1}$ for small $t \in[0, T]$ to obtain

$$
\begin{aligned}
X_{t} \approx S_{t}\left(X_{0}+t F\left(X_{0}\right)\right. \\
+\int_{0}^{t} B\left(X_{0}\right) d W_{s} \\
\left.+\int_{0}^{t} B^{\prime}\left(X_{0}\right)\left(\int_{0}^{s} B\left(X_{0}\right) d W_{u}\right) d W_{s}\right),
\end{aligned}
$$


where $S_{t}=(I-t A)^{-1}$. Combining the temporal approximation (19) and spatial discretization in (4) suggests the numerical scheme given by $Y_{0}^{N}=P_{N}(\xi)=\xi^{N}$ and

$$
\begin{aligned}
Y_{k+1}^{N}= & P_{N} S_{T / N^{2}} \\
& \times\left(Y_{k}^{N}+\frac{T}{N^{2}} F\left(Y_{k}^{N}\right)\right. \\
& +B\left(Y_{k}^{N}\right)\left(W_{(k+1) T / N^{2}}^{N}-W_{k T / N^{2}}^{N}\right) \\
& +\int_{k T / N^{2}}^{(k+1) T / N^{2}} B^{\prime}\left(Y_{k}^{N}\right) \\
& \left.\times\left(\int_{k T / N^{2}}^{s} B\left(Y_{k}^{N}\right) d W_{u}^{N}\right) d W_{s}^{N}\right),
\end{aligned}
$$

for all $k \in\left\{0,1, \ldots, N^{2}-1\right\}$ and all $N \in \mathbb{N}$, where $S_{T / N^{2}}=$ $\left(I-\left(T / N^{2}\right) A\right)^{-1}$. The difficulty in this formula is working with the term corresponding to the double integral. As suggested by Jentzen and Rockner (see [16, Subsection 6.7]), this double integral can be replaced by

$$
\begin{aligned}
\int_{k T / N^{2}}^{(k+1) T / N^{2}} B^{\prime}\left(Y_{k}^{N}\right)\left(\int_{k T / N^{2}}^{s} B\left(Y_{k}^{N}\right) d W_{u}^{N}\right) d W_{s}^{N} \\
=\frac{1}{2}\left(\frac{\partial}{\partial y} b\right)\left(\cdot, Y_{k}^{N}\right) b\left(\cdot, Y_{k}^{N}\right) \\
\quad \times\left(\left(W_{(k+1) T / N^{2}}^{N}-W_{k T / N^{2}}^{N}\right)^{2}-\frac{T}{N^{2}} \sum_{i=1}^{N} \eta_{i} g_{i}^{2}\right) .
\end{aligned}
$$

By using (21), the numerical scheme (20) thus reduces to

$$
\begin{aligned}
& Y_{k+1}^{N}= P_{N} S_{T / N^{2}}^{N} \\
& \times\left(Y_{k}^{N}+\frac{T}{N^{2}} f\left(\cdot, Y_{k}^{N}\right)+b\left(\cdot, Y_{k}^{N}\right)\right. \\
& \quad \times\left(W_{(k+1) T / N^{2}}^{N}-W_{k T / N^{2}}^{N}\right) \\
&+\frac{1}{2}\left(\frac{\partial}{\partial y} b\right)\left(\cdot, Y_{k}^{N}\right) b\left(\cdot, Y_{k}^{N}\right) \\
&\left.\quad \times\left(\left(W_{(k+1) T / N^{2}}^{N}-W_{k T / N^{2}}^{N}\right)^{2}-\frac{T}{N^{2}} \sum_{i=1}^{N} \eta_{i} g_{i}^{2}\right)\right),
\end{aligned}
$$

where $S_{T / N^{2}}^{N}=\left(I-\left(T / N^{2}\right) A\right)^{-N}$ and for all $k \in\left\{0,1, \ldots, N^{2}-\right.$ $1\}, N \in \mathbb{N}$.

For the simplified Milstein scheme (22) applied to (1), the main result of this paper, that is, Theorem 7 , will show that with $K=N^{2}$

$$
\left(E\left\|X_{T}-Y_{N^{2}}^{N}\right\|_{H}^{2}\right)^{1 / 2} \leq C_{r} N^{r-3 / 2}, \quad 0<r<\frac{3}{2} .
$$

Similar to scheme (7), the numerical method (22) can be simulated quite easily. The term $\left(T / N^{2}\right) \sum_{i=1}^{N} \eta_{i} g_{i}^{2}$ in (22) can
TABLE 1: Runtime (seconds) for one path simulation using three methods $\bar{Y}_{N^{3}}^{N}, \widetilde{Y}_{N^{2}}^{N}$, and $Y_{N^{2}}^{N}$ for $N=64,128,256,512,1024$.

\begin{tabular}{lccc}
\hline$N$ & $\begin{array}{c}\text { Implicit Euler } \\
\text { scheme }\end{array}$ & $\begin{array}{c}\text { Milstein } \\
\text { scheme }\end{array}$ & $\begin{array}{c}\text { Simplified Milstein } \\
\text { scheme }\end{array}$ \\
\hline 64 & 38.316292 & 0.703536 & 0.690610 \\
128 & 381.021714 & 3.628195 & 3.581998 \\
256 & 3874.629760 & 19.811083 & 19.405515 \\
512 & 46885.088426 & 126.663048 & 124.849040 \\
1024 & $5.1574 e+005$ & 842.699400 & 834.842365 \\
\hline
\end{tabular}

be computed once in advance for which $O\left(N^{2}\right)$ computational operations are needed. With the term $\left(T / N^{2}\right) \sum_{i=1}^{N} \eta_{i} g_{i}^{2}$ at hand, $O(N \log N)$ further computational operations and independent standard normal random variables are needed to compute $Y_{k+1}^{N}$ from $Y_{k}^{N}$ by using fast Fourier transform. Therefore, since $N^{2}$ time steps are used, $O\left(N^{3} \log N\right)$ computational operations and random variables are required to obtain $Y_{N^{2}}^{N}$. The logarithmic term in $O\left(N^{3} \log N\right)$ arises from fast Fourier transform computations, due to the nonlinearities of $f$ and $b$. Taking into account the convergence order $3 / 2$ - in (23), one can show that scheme (22) shares the same overall convergence order of $1 / 2-$, which is greater than the overall convergence order $3 / 8$ - of the linear implicit scheme (4). We then take a more closer look at schemes (7) and (22) at each step. It is obvious that the Milstein scheme (7) requires evaluation of exponential term, while the simplified Milstein scheme needs one simple mollifier $\left(I-\left(T / N^{2}\right) A\right)^{-1}$ instead of exponential term. The CPU time for one path simulation by the simplified Milstein scheme (22) applied to (1) is less than that for (7). For example, see Table 1; for $N=1024$, one path simulation of the simplified Milstein scheme (22) requires $834.842365 \mathrm{CPU}$ seconds, while this simulation by Milstein scheme (7) needs $842.699400 \mathrm{CPU}$ seconds. This difference is due to the fact that evaluation of the exponential term takes more time than that of the simple mollifier term. A natural question thus arises on whether such substitution can maintain the high convergence order of (7). In this paper we investigate this issue and prove that the simplified Milstein scheme maintains the expected order of convergence.

\section{Convergence Analysis}

Let $\left(g_{j}\right)_{j \in \mathbb{N}} \subset U$ be an orthonormal basis consisting of the eigenfunctions of $Q: U \rightarrow U$, and let $\left(\mu_{j}\right)_{j \in \mathbb{N}} \subset[0, \infty)$ be their corresponding eigenvalues with $Q: U \rightarrow U$ as a trace class operator; that is,

$$
\mathrm{Q} u=\sum_{j \in \mathbb{N}} \mu_{j}\left\langle g_{j}, u\right\rangle_{U} g_{j}
$$

for all $u \in U$. We define the linear projection operator

$$
P_{N}: H \longrightarrow H \quad \text { by } P_{N} v=\sum_{j=1}^{N}\left\langle e_{j}, v\right\rangle_{H} e_{j} .
$$

Furthermore, we define Wiener processes $W^{N}:[0, T] \times \Omega \rightarrow$ $U_{0}$ by $W_{t}^{N}(\omega)=\sum_{j=1}^{N}\left\langle g_{j}, W_{t}(\omega)\right\rangle_{U} g_{j}$ for all $t \in[0, T]$, 
$\omega \in \Omega$, and $N \in \mathbb{N}$. Let $T=M \Delta t, M=N^{2}$, and $\Delta t$ be the time discretization step, and let $H=U=L^{2}((0,1), \mathbb{R})$ be the $\mathbb{R}$-Hilbert space of equivalence classes of $\beta(0,1) / \beta(\mathbb{R})$ measurable and Lebesgue square integrable functions from $(0,1)$ to $\mathbb{R}$ with the scalar product $\langle u, v\rangle_{H}=\int_{0}^{1} u(x) v(x) d x$ and the norm $\|v\|_{H}=\left(\int_{0}^{1}|v(x)|^{2} d x\right)^{1 / 2}$ for $u, v \in H=U$. Now we start our investigation to analyze the proposed method for SPDE fulfilling Assumptions 1-4. Based on (1) we then consider $A=\lambda\left(\partial^{2} / \partial x^{2}\right)$ and $e_{k}(x)=\sqrt{2} \sin (k \pi x), k \in \mathbb{N}$, as the orthonormal basis of $H=L^{2}((0,1), \mathbb{R})$, which satisfy

$$
A e_{k}=-\lambda_{k} e_{k}, \quad \lambda_{k}=\lambda \pi^{2} k^{2} .
$$

For the drift term to fulfill Assumption 2, let $f:(0,1) \times$ $\mathbb{R} \rightarrow \mathbb{R}$ be a continuously differentiable function with $\int_{0}^{1}|f(x, 0)|^{2} d x<\infty$ and

$$
\sup _{y \in \mathbb{R}}\left|\frac{\partial f}{\partial y}(x, y)\right|<\infty .
$$

Then, the operator $F: H \rightarrow H$ given by $(F(v))(x)=$ $f(x, v(x))$, for $x \in(0,1)$ and $v \in H$, satisfies Assumption 2 . For the diffusion term to satisfy Assumption 3, we consider $b:(0,1) \times \mathbb{R} \rightarrow \mathbb{R}$ to be a twice continuously differentiable function with

$$
\begin{gathered}
|b(x, 0)| \leq R, \quad\left|\frac{\partial b}{\partial x}(x, y)\right| \leq R, \\
\left\|\frac{\partial b}{\partial x}(x, y)\right\|_{L(\mathbb{R}, \mathbb{R})} \leq R,
\end{gathered}
$$

and also

$$
\begin{gathered}
E\left\|B\left(X_{t}\right)\right\|_{H S\left(U_{0}, V_{\delta}\right)}^{2} \leq R, \quad\left\|F^{\prime}(v)\right\|_{L(H)} \leq R, \\
\left\|F^{\prime \prime}(v)\right\|_{L^{(2)}\left(V_{\beta}, H\right)} \leq R, \quad E\left\|F\left(X_{t}\right)\right\|_{H}^{2} \leq R, \\
\left\|B^{\prime}(v)\right\|_{L\left(H, H S\left(U_{0}, V_{\delta}\right)\right)} \leq R, \quad\left\|B^{\prime \prime}(v)\right\|_{L^{(2)}\left(V_{\beta}, H S\left(U_{0}, V_{\delta}\right)\right)} \leq R, \\
E\left\|(-A)^{\gamma} X_{t}\right\|_{H}^{2}=E\left\|X_{t}\right\|_{V_{\gamma}}^{2} \leq R, \\
E\left\|X_{t_{2}}-X_{t_{1}}\right\|_{V_{\beta}}^{4} \leq R\left|t_{2}-t_{1}\right|^{\min (4(\gamma-\beta), 2)},
\end{gathered}
$$

for all $x \in(0,1)$ and some given $R \in(0, \infty)$. Let $b: H \rightarrow$ $H S\left(U_{0}, H\right)$ be the operator $(B(v) u)(x)=b(x, v(x)) \cdot u(x)$ for all $x \in(0,1)$.

It has been shown in [16] that $B: H \rightarrow H S\left(U_{0}, H\right)$ fulfills Assumption 3. For the initial value to satisfy Assumption 4, we assume that $x_{0}:(0,1) \rightarrow \mathbb{R}$ is a twice continuously differentiable function with $\left.x_{0}\right|_{\partial(0,1)}=0$. Then the mapping $\xi: \Omega \rightarrow V_{\gamma}$ given by $\xi(\omega) \stackrel{x_{0}}{=} x_{0}$ for all $\omega \in \Omega$ fulfills Assumption 4 for all $\gamma \in(0,1)$. With the above setting, the SPDE (1) reduces to

$$
\begin{aligned}
d X_{t}(x)= & {\left[\lambda \Delta X_{t}(x)+f\left(x, X_{t}(x)\right)\right] d t } \\
& +b\left(x, X_{t}(x)\right) d W_{t}(x),
\end{aligned}
$$

with $X_{t}(0)=X_{t}(1)=0$ and $X_{0}=x_{0}(x)$ for $t \in[0, T], x \in$ $(0,1)$, and $\Delta=\partial^{2} / \partial x^{2}$.

Moreover, we define a family $\beta^{j}(\omega)=\left(1 / \sqrt{\mu_{j}}\right)$ $\left\langle g_{j}, W_{t}(\omega)\right\rangle_{U}$ for all $\omega \in \Omega, t \in[0, T]$ and all $j \in \mathbb{N}$, and we consider the mappings $\Delta W_{k}^{N}: \Omega \rightarrow U_{0}, k \in\left\{0,1, \ldots, N^{2}-1\right\}$ by $\Delta W_{k}^{N}(\omega)=W_{(k+1) \Delta t}^{N}(\omega)-W_{k \Delta}^{N}(\omega)$.

Using these notations, the SPDE (30) can be rewritten as

$$
\begin{aligned}
d X_{t}(x)= & {\left[\lambda \frac{\partial}{\partial x^{2}} X_{t}(x)+f\left(x, X_{t}(x)\right)\right] d t } \\
& +\sum_{j=1}^{N}\left[b\left(x, X_{t}(x)\right) \sqrt{\mu_{j}} g_{j}(x)\right] d \beta_{t}^{j},
\end{aligned}
$$

with $X_{t}(0)=X_{t}(1)=0$ and $X_{0}(x)=x_{0}(x)$ for $t \in[0, T]$ and $x \in(0,1)$.

Scheme (21)-(22) applied to the SPDE (30) reduces to

$$
\begin{aligned}
Y_{k+1}^{N}=P_{N}( & S_{\Delta t} Y_{k}^{N}+\Delta t S_{\Delta t} F\left(Y_{k}^{N}\right) \\
& +\int_{k \Delta t}^{(k+1) \Delta t} S_{\Delta t} B\left(Y_{k}^{N}\right) d W_{s}^{N} \\
& +\int_{k \Delta t}^{(k+1) \Delta t} S_{\Delta t} B^{\prime}\left(Y_{k}^{N}\right) \\
& \left.\times\left(\int_{k \Delta t}^{s} B\left(Y_{k}^{N}\right) d W_{u}^{N}\right) d W_{s}^{N}\right),
\end{aligned}
$$

where $S_{\Delta t}=(I-\Delta t A)^{-1}$ and $\Delta t=T / N^{2}, k \in\left\{0,1, \ldots, N^{2}-1\right\}$, $N \in \mathbb{N}$. Therefore the numerical method (32) satisfies

$$
\begin{aligned}
Y_{k}^{N}= & S_{\Delta t}^{k} Y_{0}^{N}+P_{N}\left(\sum_{l=0}^{k-1} \int_{l \Delta t}^{(l+1) \Delta t} S_{\Delta t}^{k-l} F\left(Y_{l}^{N}\right) d s\right) \\
& +P_{N}\left(\sum_{l=0}^{k-1} \int_{l \Delta t}^{(l+1) \Delta t} S_{\Delta t}^{k-l} B\left(Y_{l}^{N}\right) d W_{s}^{N}\right) \\
& +P_{N}\left(\sum_{l=0}^{k-1} \int_{l \Delta t}^{(l+1) \Delta t} S_{\Delta t}^{k-l} B^{\prime}\left(Y_{l}^{N}\right)\right. \\
& \left.\times\left(\int_{l \Delta t}^{s} B\left(Y_{l}^{N}\right) d W_{u}^{N}\right) d W_{s}^{N}\right),
\end{aligned}
$$

where $S_{\Delta t}^{k}=(I-\Delta t A)^{-k}$ and for all $k \in\left\{0,1, \ldots, N^{2}\right\}, N \in$ $\mathbb{N}$. The following inequalities are classical and one can easily prove them by using the spectral decomposition of $A[1]$ :

$$
\begin{gathered}
\left\|(-A)^{s} S_{\Delta t}^{l}\right\|_{L(H)} \leq M t_{l}^{-s}, \quad l \geq 1, \quad s \in[0,1], \\
\left\|(-A)^{s} e^{t A}\right\|_{L(H)} \leq M t^{-s}, \quad t>0, \\
\left\|S_{\Delta t}^{l}-e^{l \Delta t A}\right\|_{L(H)} \leq \frac{M}{l}, \\
\left\|(-A)^{-s}\left(e^{t A}-I\right)\right\|_{L(H)} \leq M t^{s}, \\
\left\|(-A)^{-s}\left(S_{\Delta t}-I\right)\right\|_{L(H)} \leq M(\Delta t)^{s}, \quad s \in[0,1] .
\end{gathered}
$$


To give the order of the $L^{2}$ convergence for the simplified Milstein approximation of the evolution equation, we need the following version of the Gronwall lemma.

Lemma 6. Let $\left\{\alpha_{n}\right\}_{n \geq 0},\left\{\beta_{n}\right\}_{n \geq 0}$ be two sequences of nonnegative numbers such that $\alpha_{0}=\beta_{0}=0$ and such that there exists a positive constant $L$ such that

$$
\alpha_{n} \leq L \sum_{k=0}^{n-1} \alpha_{k}+\beta_{n}, \quad \forall n \geq 1
$$

then

$$
\alpha_{n} \leq \sum_{k=0}^{n-1} e^{(n-k-1) L}\left(\beta_{(k+1)}-\beta_{k}\right), \quad \forall n \geq 1 .
$$

Proof. By Mathematical induction with respect to $n$ using $L \leq$ $e^{L}-1$.

From the above lemma we can deduce that

$$
\forall n \geq 1, \quad \alpha_{n} \leq e^{(n-1) L} \beta_{n} .
$$

The main result of this section is stated below.

Theorem 7. Let $T>0, \Delta t=T / N^{2}$, and $X_{0} \in L^{2}(\Omega, H)$. Suppose that $X$ is the solution of (2) on $[0, T]$. Let Assumptions 1-4 hold, and let $\left\{Y_{l}^{N}\right\}_{l \geq 0}$ be the numerical approximations obtained by scheme (33). Then there exists a positive constant C such that

$$
\begin{aligned}
& \left(E\left\|e_{k}^{N}\right\|_{H}^{2}\right)^{1 / 2} \\
& \leq C\left(\left(\inf _{j>N+1} \lambda_{j}\right)^{-\gamma}+\frac{\left(E\left\|X_{0}\right\|_{H}^{2}\right)^{1 / 2}}{k}\right. \\
& \left.\quad+\left(\sup _{j>N+1} \mu_{j}\right)^{\alpha}+(\Delta t)^{\min (2(\gamma-\beta), \gamma)}\right) .
\end{aligned}
$$

Proof. To start the proof, we first note that the exact solution of SPDE (2) satisfies

$$
\begin{aligned}
X_{k \Delta t}= & e^{A t} X_{0}+\int_{0}^{k \Delta t} e^{A(t-s)} F\left(X_{s}\right) d s \\
& +\int_{0}^{k \Delta t} e^{A(t-s)} B\left(X_{s}\right) d W_{s} \\
= & e^{A t} X_{0}+\sum_{l=0}^{k-1} \int_{l \Delta t}^{(l+1) \Delta t} e^{A(t-s)} F\left(X_{s}\right) d s \\
& +\sum_{l=0}^{k-1} \int_{l \Delta t}^{(l+1) \Delta t} e^{A(t-s)} B\left(X_{s}\right) d W_{s},
\end{aligned}
$$

where $t=k \Delta t$. In particular, (43) shows

$$
\begin{aligned}
P_{N}\left(X_{k \Delta t}\right)= & e^{A t} X_{0}^{N}+P_{N}\left(\int_{0}^{k \Delta t} e^{A(t-s)} F\left(X_{s}\right) d s\right) \\
& +P_{N}\left(\int_{0}^{k \Delta t} e^{A(t-s)} B\left(X_{s}\right) d W_{s}\right) \\
= & e^{A t} X_{0}^{N}+P_{N}\left(\sum_{l=0}^{k-1} \int_{l \Delta t}^{(l+1) \Delta t} e^{A(t-s)} F\left(X_{s}\right) d s\right) \\
& +P_{N}\left(\sum_{l=0}^{k-1} \int_{l \Delta t}^{(l+1) \Delta t} e^{A(t-s)} B\left(X_{s}\right) d W_{s}\right) .
\end{aligned}
$$

Let

$$
\begin{aligned}
e_{k}^{N} & =X_{k \Delta t}-Y_{k}^{N} \\
& =X_{k \Delta t}-P_{N}\left(X_{k \Delta t}\right)+P_{N}\left(X_{k \Delta t}\right)-Y_{k}^{N} \\
& =\bar{e}_{k}^{N}+\widehat{e}_{k}^{N},
\end{aligned}
$$

where

$$
\bar{e}_{k}^{N}=X_{k \Delta t}-P_{N}\left(X_{k \Delta t}\right), \quad \hat{e}_{k}^{N}=P_{N}\left(X_{k \Delta t}\right)-Y_{k}^{N} .
$$

For the spatial discretization error $\bar{e}_{k}^{N}$, we have

$$
\begin{aligned}
E\left\|\bar{e}_{k}^{N}\right\|_{H}^{2} & =E\left\|X_{k \Delta t}-P_{N}\left(X_{k \Delta t}\right)\right\|_{H}^{2} \\
& =E\left\|\left(I-P_{N}\right) X_{k \Delta t}\right\|_{H}^{2} \\
& =E\left\|(-A)^{-\gamma}\left(I-P_{N}\right)(-A)^{\gamma} X_{k \Delta t}\right\|_{H}^{2} \\
& \leq\left\|(-A)^{-\gamma}\left(I-P_{N}\right)\right\|_{L(H)}^{2} E\left\|X_{k \Delta t}\right\|_{V_{\gamma}}^{2} \\
& \leq R\left(s_{N}\right)^{2} ;
\end{aligned}
$$

the real numbers $\left(s_{N}\right)_{N \in \mathbb{N}}$ are given by (see [16])

$$
s_{N}:=\left\|(-A)^{-\gamma}\left(I-P_{N}\right)\right\|_{L(H)}=\left(\inf _{j>N+1} \lambda_{j}\right)^{-\gamma} .
$$

For the $\hat{e}_{k}^{N}$ with respect to (33) and (44), we have

$$
\begin{aligned}
\hat{e}_{k}^{N}= & e^{A t} X_{0}^{N}-S_{\Delta t}^{k} Y_{0}^{N} \\
& +P_{N}\left(\sum _ { l = 0 } ^ { k - 1 } \int _ { l \Delta t } ^ { ( l + 1 ) \Delta t } \left(e^{A(t-s)} F\left(X_{s}\right)\right.\right. \\
& \left.\left.-s_{\Delta t}^{k-l} F\left(Y_{l}^{N}\right)\right) d s\right) \\
& +P_{N}\left(\sum _ { l = 0 } ^ { k - 1 } \int _ { l \Delta t } ^ { ( l + 1 ) \Delta t } \left(e^{A(t-s)} B\left(X_{s}\right) d W_{s}\right.\right.
\end{aligned}
$$




$$
\begin{gathered}
\left.-S_{\Delta t}^{k-l} B\left(Y_{l}^{N}\right) d W_{s}^{N}\right) \\
-\sum_{l=0}^{k-1} \int_{l \Delta t}^{(l+1) \Delta t} S_{\Delta t}^{k-l} B^{\prime}\left(Y_{l}^{N}\right) \\
\left.\quad \times\left(\int_{l \Delta t}^{s} B\left(Y_{l}^{N}\right) d W_{u}^{N}\right) d W_{s}^{N}\right)
\end{gathered}
$$$$
=I+I I+I I I,
$$

where $I=e^{A t} X_{0}^{N}-S_{\Delta t}^{k} Y_{0}^{N}$ and $I I$ and $I I I$ are, respectively, the other terms under $P_{N}$ operator. From (36), the first term of (49) can be easily estimated by

$$
\begin{aligned}
& E\|I\|_{H}^{2} \\
& \quad=\left(E\left\|\left(e^{A t}-S_{\Delta t}^{k}\right) X_{0}^{N}+S_{\Delta t}^{k}\left(X_{0}^{N}-Y_{0}^{N}\right)\right\|_{H}^{2}\right) \\
& \quad \leq C\left(\frac{E\left\|X_{0}^{N}\right\|_{H}^{2}}{k^{2}}+E\left\|\hat{e}_{0}^{N}\right\|_{H}^{2}\right) .
\end{aligned}
$$

Let $C$ denote a constant which may depend on $A, f, b, R, N$, $M$, or $T$. We now treat the second term of (49)

$$
\begin{aligned}
I I= & P_{N}\left(\sum _ { l = 0 } ^ { k - 1 } \int _ { l \Delta t } ^ { ( l + 1 ) \Delta t } \left(e^{A(t-s)} F\left(X_{s}\right)\right.\right. \\
& \left.\left.-S_{\Delta t}^{k-l} F\left(Y_{l}^{N}\right)\right) d s\right) \\
= & P_{N}\left(\sum_{l=0}^{k-1} \int_{l \Delta t}^{(l+1) \Delta t}\left(e^{A(t-s)}-S_{\Delta t}^{k-l}\right) F\left(X_{s}\right) d s\right) \\
& +P_{N}\left(\sum_{l=0}^{k-1} \int_{l \Delta t}^{(l+1) \Delta t} S_{\Delta t}^{k-l}\left(F\left(X_{s}\right)-F\left(X_{l \Delta t}\right)\right) d s\right) \\
& +P_{N}\left(\sum_{l=0}^{k-1} \int_{l \Delta t}^{(l+1) \Delta t} S_{\Delta t}^{k-l}\left(F\left(X_{l \Delta t}\right)-F\left(Y_{l}^{N}\right)\right) d s\right) \\
= & I I_{1}+I I_{2}+I I_{3} .
\end{aligned}
$$

According to Assumption 2 and the fact that $\left\|P_{N}(v)\right\|_{H} \leqslant$ $\|v\|_{H}$ for all $v \in H$, we get

$$
\begin{gathered}
\left\|I I_{1}\right\|_{H} \leq C \sum_{l=0}^{k-1} \int_{l \Delta t}^{(l+1) \Delta t}\left\|e^{A(t-s)}-S_{\Delta t}^{k-l}\right\|_{L(H)} \\
\times\left(1+\left\|X_{s}\right\|_{V_{\gamma}}\right) d s
\end{gathered}
$$

$$
\begin{gathered}
\leq C \int_{(k-1) \Delta t}^{k \Delta t}\left\|e^{A(t-s)}-S_{\Delta t}\right\|_{L(H)}\left(1+\left\|X_{s}\right\|_{V_{\gamma}}\right) d s \\
+C \sum_{l=0}^{k-2} \int_{l \Delta t}^{(l+1) \Delta t}\left\|e^{A(t-s)}-s_{\Delta t}^{k-l}\right\|_{L(H)} \\
\times\left(1+\left\|X_{s}\right\|_{V_{\gamma}}\right) d s .
\end{gathered}
$$

For the first term on the right-hand side of inequality (52), we have

$$
\begin{aligned}
& E\left(\int_{(k-1) \Delta t}^{k \Delta t}\left\|e^{A(t-s)}-S_{\Delta t}\right\|_{L(H)}\left(1+\left\|X_{s}\right\|_{V_{\gamma}}\right) d s\right)^{2} \\
& \quad \leq M(\Delta t)^{2}\left(1+\sup _{t \in[0, T]} E\left\|X_{t}\right\|_{V_{\gamma}}^{2}\right) .
\end{aligned}
$$

To estimate the second term of (52), one should note that

$$
\begin{aligned}
\left\|e^{A(t-s)}-S_{\Delta t}^{k-l}\right\|_{L(H)} \leq & \left\|e^{A(t-s)}-e^{A(k-l-1) \Delta t}\right\|_{L(H)} \\
& +\left\|e^{A(k-l-1) \Delta t}-S_{\Delta t}^{k-l}\right\|_{L(H)}
\end{aligned}
$$

and from (35) and (37), we have

$$
\begin{aligned}
\left\|e^{A(t-s)}-e^{A(k-l-1) \Delta t}\right\|_{L(H)} \leq & \left\|(-A)^{\gamma} e^{A(k-l-1) \Delta t}\right\|_{L(H)} \\
& \times\left\|(-A)^{-\gamma}\left(e^{A(l+1) \Delta t-s}-I\right)\right\|_{L(H)} \\
\leq & C \frac{((l+1) \Delta t-s)^{\gamma}}{((k-l-1) \Delta t)^{\gamma}} .
\end{aligned}
$$

Therefore, from (35), (38), and (36), we have

$$
\begin{aligned}
&\left\|e^{A(k-l-1) \Delta t}-S_{\Delta t}^{k-l}\right\|_{L(H)} \\
& \leq\left\|(-A)^{\gamma}\left(e^{A(k-l-1) \Delta t}\right)(-A)^{-\gamma}\left(I-S_{\Delta t}\right)\right\|_{L(H)} \\
&+\left\|S_{\Delta t}\left(e^{A(k-l-1) \Delta t}-S_{\Delta t}^{k-l-1}\right)\right\|_{L(H)} \\
& \leq M\left(\frac{\Delta t^{\gamma}}{((k-l-1) \Delta t)^{\gamma}}+\frac{1}{k-l-1}\right) \\
& \leq C \frac{(\Delta t)^{\gamma}}{((k-l-1) \Delta t)^{\gamma}} ;
\end{aligned}
$$

thus we have

$$
\left\|e^{A(t-s)}-S_{\Delta t}^{k-l}\right\|_{L(H)} \leq M\left(\frac{(\Delta t)^{\gamma}}{((k-l-1) \Delta t)^{\gamma}}\right) .
$$


Therefore, by taking the expectation of (52) to the power of 2 and using (53) and (57), we get

$$
\begin{aligned}
& E\left\|I I_{1}\right\|_{H}^{2} \\
& \leq C\left(\sum_{l=0}^{k-2} \int_{l \Delta t}^{(l+1) \Delta t} \frac{(\Delta t)^{\gamma}}{((k-l-1) \Delta t)^{\gamma}}\left(1+E\left\|X_{s}\right\|_{V_{\gamma}}^{2}\right) d s\right)^{2} \\
& \quad+C(\Delta t)^{2}
\end{aligned}
$$

in which the summation can be estimated as

$$
\begin{aligned}
\sum_{l=0}^{k-2} \frac{\Delta t}{((k-l-1) \Delta t)^{d}} & \leq \sum_{l=0}^{k-1} \int_{l \Delta t}^{(l+1) \Delta t} \frac{d t}{t^{d}} \\
& \leq \frac{1}{1-d} T^{1-d}, \quad d<1 .
\end{aligned}
$$

For the second term of (51), Proposition 5 and Assumption 2 lead to

$$
\begin{aligned}
E\left\|I I_{2}\right\|_{H}^{2} & \leq \sum_{l=0}^{k-1} \int_{l \Delta t}^{(l+1) \Delta t}\left\|S_{\Delta t}^{k-l}\right\|_{L(H)}^{2}(\Delta t)^{2 \min \{\gamma, 1 / 2\}} d s \\
& \leq C(\Delta t)^{2 \min \{\gamma, 1 / 2\}} .
\end{aligned}
$$

For the third term of (51), by using Assumption 2, we have

$$
\begin{aligned}
\left\|I I_{3}\right\|_{H} & \leq \sum_{l=0}^{k-1} \int_{l \Delta t}^{(l+1) \Delta t}\left\|S_{\Delta t}^{k-l}\left(f\left(X_{l \Delta t}\right)-f\left(Y_{l}^{N}\right)\right)\right\|_{H} d s \\
& \leq \sum_{l=0}^{k-1} \int_{l \Delta t}^{(l+1) \Delta t}\left\|S_{\Delta t}^{k-l}\right\|_{L(H)}\left\|e_{l}^{N}\right\|_{H} d s \\
& \leq C \sum_{l=0}^{k-1} \Delta t\left\|e_{l}^{N}\right\|_{H}
\end{aligned}
$$

which leads to

$$
E\left\|I I_{3}\right\|_{H}^{2} \leq C\left(\sum_{l=0}^{k-1}(\Delta t)\left(E\left\|e_{l}^{N}\right\|_{H}^{2}\right)^{1 / 2}\right)^{2}
$$

where we have used the Minkowski inequality in (62).

Therefore, from (58), (60), and (62), we get

$$
\begin{array}{r}
E\|I I\|_{H}^{2} \leq C\left((\Delta t)^{2 \gamma}+(\Delta t)^{2 \min \{\gamma, 1 / 2\}}\right. \\
\left.+\left(\sum_{l=0}^{k-1}(\Delta t)\left(E\left\|e_{l}^{N}\right\|_{H}^{2}\right)^{1 / 2}\right)^{2}\right) .
\end{array}
$$

Now for the last term of (49), we obtain

$$
\begin{gathered}
I I I=P_{N}\left(\sum _ { l = 0 } ^ { k - 1 } \int _ { l \Delta t } ^ { ( l + 1 ) \Delta t } \left(e^{A(t-s)} B\left(X_{s}\right) d W_{s}\right.\right. \\
\left.\quad-S_{\Delta t}^{k-l} B\left(Y_{l}^{N}\right) d W_{s}^{N}\right) \\
\quad-\sum_{l=0}^{k-1} \int_{l \Delta t}^{(l+1) \Delta t} S_{\Delta t}^{k-l} B^{\prime}\left(Y_{l}^{N}\right) \\
\left.\times\left(\int_{l \Delta t}^{s} B\left(Y_{l}^{N}\right) d W_{u}^{N}\right) d W_{s}^{N}\right) \\
=I I I_{1}+I I I_{2}+I I I_{3}+I I I_{4},
\end{gathered}
$$

where

$$
\left.\begin{array}{c}
I I I_{1}=P_{N}\left(\sum_{l=0}^{k-1} \int_{l \Delta t}^{(l+1) \Delta t} e^{A(t-s)}\right. \\
\left.\times B\left(X_{s}\right)\left(d W_{s}-d W_{s}^{N}\right)\right), \\
I I I_{2}=P_{N}\left(\sum_{l=0}^{k-1} \int_{l \Delta t}^{(l+1) \Delta t}\left(e^{A(t-s)}-S_{\Delta t}^{k-l}\right)\right. \\
\left.\times I_{N}\left(\sum_{l=0}^{k-1} \int_{l \Delta t}^{(l+1) \Delta t} S_{\Delta t}^{k-l} X_{s}\right) d W_{s}^{N}\right), \\
\left.I I I_{4}=P_{N}\left(\sum_{l=0}^{k-1} \int_{l \Delta t}^{(l+1) \Delta t} S_{\Delta t}^{k-l}\left(X_{l \Delta t}\right)-B\left(Y_{l}^{N}\right)\right) d W_{s}^{N}\right) \\
-\sum_{l=0}^{k-1} \int_{l \Delta t}^{(l+1) \Delta t} S_{\Delta t}^{k-l} B^{\prime}\left(Y_{l}^{N}\right) \\
\left.\left.\times\left(X_{l \Delta t}^{s} B\left(Y_{l}^{N}\right) d W_{u}^{N}\right) d W_{s}^{N}\right) . B\left(X_{l \Delta t}\right)\right) d W_{s}^{N}
\end{array}\right) .
$$

Using the fact that $\left\|P_{N}(v)\right\|_{H} \leqslant\|v\|_{H}$ for all $v \in H$, for $I I I_{1}$, we have

$$
\begin{aligned}
& E\left\|\int_{0}^{t} e^{A(t-s)} B\left(X_{s}\right) d\left(W_{s}-W_{s}^{N}\right)\right\|_{H}^{2} \\
& =E\left\|\sum_{j=N+1}^{\infty}\left(\int_{0}^{t} e^{A(t-s)} B\left(X_{s}\right) g_{j} d\left\langle g_{j}, W_{s}\right\rangle\right)\right\|_{H} \\
& =\sum_{j=N+1}^{\infty} \mu_{j}\left(\int_{0}^{t} E\left\|e^{A(t-s)} B\left(X_{s}\right) g_{j}\right\|_{H}^{2} d s\right),
\end{aligned}
$$


and therefore

$$
\begin{aligned}
& E\left\|\int_{0}^{t} e^{A(t-s)} B\left(X_{s}\right) d\left(W_{s}-W_{s}^{N}\right)\right\|_{H}^{2} \\
& =\sum_{j=N+1}^{\infty} \mu_{j}\left(\int_{0}^{t} E\left\|e^{A(t-s)} B\left(X_{s}\right) Q^{-\alpha} Q^{\alpha} g_{j}\right\|_{H}^{2} d s\right) \\
& =\sum_{j=N+1}^{\infty}\left(\mu_{j}\right)^{2 \alpha+1}\left(\int_{0}^{t} E\left\|e^{A(t-s)} B\left(X_{s}\right) Q^{-\alpha} g_{j}\right\|_{H}^{2} d s\right) .
\end{aligned}
$$

Thus

$$
\begin{aligned}
E\left\|\int_{0}^{t} e^{A(t-s)} B\left(X_{s}\right) d\left(W_{s}-W_{s}^{N}\right)\right\|_{H}^{2} \\
\leq\left(\sup _{j>N+1} \mu_{j}\right) \\
\quad \times \sum_{j=N+1}^{\infty} \mu_{j}\left(\int_{0}^{t} E\left\|e^{A(t-s)} B\left(X_{s}\right) Q^{-\alpha} g_{j}\right\|_{H}^{2} d s\right) \\
\leq\left(\sup _{j>N+1} \mu_{j}\right)^{2 \alpha} \\
\quad \times \sum_{j=1}^{\infty} \mu_{j}\left(\int_{0}^{t} E\left\|e^{A(t-s)} B\left(X_{s}\right) Q^{-\alpha} g_{j}\right\|_{H}^{2} d s\right)
\end{aligned}
$$

which means

$$
\begin{aligned}
E\left\|\int_{0}^{t} e^{A(t-s)} B\left(X_{s}\right) d\left(W_{s}-W_{s}^{N}\right)\right\|_{H}^{2} \\
\leq\left(\sup _{j>N+1} \mu_{j}\right)^{2 \alpha} \\
\quad \times\left(\int_{0}^{t} E\left\|e^{A(t-s)} B\left(X_{s}\right) Q^{-\alpha} g_{j}\right\|_{H S\left(U_{0}, H\right)}^{2} d s\right) \\
\leq\left(\sup _{j>N+1} \mu_{j}\right)^{2 \alpha} \\
\quad \times\left(\int_{0}^{t}(t-s)^{2 \theta} E\left\|(-A)^{-\theta} B\left(X_{s}\right) Q^{-\alpha}\right\|_{H S\left(U_{0}, H\right)}^{2} d s\right) .
\end{aligned}
$$

Therefore, from (13), we get

$$
\begin{aligned}
& E\left\|\int_{0}^{t} e^{A(t-s)} B\left(X_{s}\right) d\left(W_{s}-W_{s}^{N}\right)\right\|_{H}^{2} \\
& \leq C\left(\sup _{j>N+1} \mu_{j}\right)^{2 \alpha} \\
& \quad \times\left(\int_{0}^{t}(t-s)^{-2 \theta} E\left[\left(1+\left\|X_{s}\right\|_{V_{\gamma}}\right)^{2}\right] d s\right) \\
& \leq C\left(\sup _{j>N+1} \mu_{j}\right)^{2 \alpha}
\end{aligned}
$$

$$
\begin{aligned}
& \times\left(\int_{0}^{t}(t-s)^{-2 \theta}\left[\left(1+E\left\|X_{s}\right\|_{V_{\gamma}}\right)^{2}\right] d s\right) \\
\leq & C\left(\sup _{j>N+1} \mu_{j}\right)^{2 \alpha}\left(\int_{0}^{t}(t-s)^{-2 \theta} d s\right),
\end{aligned}
$$

which leads to

$$
\begin{aligned}
E\left\|I I I_{1}\right\|_{H}^{2} & \leq E\left\|\int_{0}^{t} e^{A(t-s)} B\left(X_{s}\right) d\left(W_{s}-W_{s}^{N}\right)\right\|_{H}^{2} \\
& \leq C\left(\sup _{j>N+1} \mu_{j}\right)^{2 \alpha} .
\end{aligned}
$$

For $\mathrm{III}_{2}$, by Proposition 5, it is seen that

$$
\begin{aligned}
& E\left\|\sum_{l=0}^{k-1} \int_{l \Delta t}^{(l+1) \Delta t}\left(e^{A(t-s)}-S_{\Delta t}^{k-l}\right) B\left(X_{s}\right) d W_{s}^{N}\right\|_{H}^{2} \\
& \leq \sum_{l=0}^{k-1} \int_{l \Delta t}^{(l+1) \Delta t} E\left\|\left(e^{A(t-s)}-S_{\Delta t}^{k-l}\right) B\left(X_{s}\right)\right\|_{H S\left(U_{0}, H\right)}^{2} d s \\
& \leq \sum_{l=0}^{k-1} \int_{l \Delta t}^{(l+1) \Delta t}\left\|(-A)^{-\delta}\left(e^{A(t-s)}-S_{\Delta t}^{k-l}\right)\right\|_{L(H)}^{2} \\
& \leq R \sum_{l=0}^{k-1} \int_{l \Delta t}^{(l+1) \Delta t}\left\|(-A)^{-\delta}\left(e^{A(t-s)}-S_{\Delta t}^{k-l}\right)\right\|_{L(H)}^{2} d s,
\end{aligned}
$$

in which

$$
\begin{aligned}
& \sum_{l=0}^{k-1} \int_{l \Delta t}^{(l+1) \Delta t}\left\|(-A)^{-\delta}\left(e^{A(t-s)}-S_{\Delta t}^{k-l}\right)\right\|_{L(H)}^{2} d s \\
& =\sum_{l=0}^{k-2} \int_{l \Delta t}^{(l+1) \Delta t}\left\|(-A)^{-\delta}\left(e^{A(t-s)}-S_{\Delta t}^{k-l}\right)\right\|_{L(H)}^{2} d s \\
& \quad+\int_{(k-1) \Delta t}^{k \Delta t}\left\|(-A)^{-\delta}\left(e^{A(t-s)}-S_{\Delta t}\right)\right\|_{L(H)}^{2} d s .
\end{aligned}
$$

Now for the first term on the right-hand side of (73), we need to estimate

$$
\begin{aligned}
\left\|(-A)^{-\delta}\left(e^{A(t-s)}-S_{\Delta t}^{k-l}\right)\right\|_{L(H)}^{2} \\
\leq\left\|(-A)^{-\delta}\left(e^{A(t-s)}-e^{A(k-l-1) \Delta t}\right)\right\|_{L(H)} \\
\quad+\left\|(-A)^{-\delta}\left(e^{A(k-l-1) \Delta t}-S_{\Delta t}^{k-l}\right)\right\|_{L(H)}
\end{aligned}
$$


and then by (34) and (36), we obtain

$$
\begin{aligned}
\left\|(-A)^{-\delta}\left(e^{A(t-s)}-e^{A(k-l-1) \Delta t}\right)\right\|_{L(H)} \\
\leq\left\|(-A)^{1-\delta} e^{A(k-l-1) \Delta t}\right\|_{L(H)} \\
\times\left\|(-A)^{-1}\left(e^{A(l+1) \Delta t-s}-I\right)\right\|_{L(H)} \\
\leq C \frac{((k+1) \Delta t-s)}{((k-l-1) \Delta t)^{1-\delta}} .
\end{aligned}
$$

Similarly from (34), (35), we obtain

$$
\begin{aligned}
&\left\|(-A)^{-\delta}\left(e^{A(k-l-1) \Delta t}-S_{\Delta t}^{k-l}\right)\right\|_{L(H)} \\
& \leq\left\|(-A)^{1 / 2-\delta} e^{A(k-l-1) \Delta t}(-A)^{-1 / 2}\left(I-S_{\Delta t}\right)\right\|_{L(H)} \\
& \quad+\left\|(-A)^{-\delta} S_{\Delta t}\left(e^{A(k-l-1) \Delta t}-S_{\Delta t}^{k-l-1}\right)\right\|_{L(H)} \\
& \leq C\left(\frac{(\Delta t)^{1 / 2}}{((k-l-1) \Delta t)^{1 / 2-\delta}}+\frac{1}{k-l-1}\right) \\
& \leq C\left(\frac{(\Delta t)^{1 / 2}}{((k-l-1) \Delta t)^{1 / 2-\delta}}+\frac{(\Delta t)^{\gamma_{1}}}{((k-l-1) \Delta t)^{\gamma_{1}}}\right),
\end{aligned}
$$

where $\gamma_{1}>0$ is such that

$$
\tilde{\gamma}<\gamma_{1}<\frac{1}{2}
$$

which is possible since

$$
\tilde{\gamma}<\frac{1}{2}
$$

Therefore,

$$
\begin{aligned}
& \left\|(-A)^{-\delta}\left(e^{A(t-s)}-S_{\Delta t}^{k-l}\right)\right\|_{L(H)}^{2} \\
& \quad \leq C\left(\frac{(\Delta t)}{((k-l-1) \Delta t)^{1-2 \delta}}+\frac{(\Delta t)^{2 \gamma_{1}}}{((k-l-1) \Delta t)^{2 \gamma_{1}}}\right) .
\end{aligned}
$$

For the second term on the right-hand side of (73), we can write

$$
\begin{aligned}
& \left\|(-A)^{-\delta}\left(e^{A(t-s)}-S_{\Delta t}\right)\right\|_{L(H)}^{2} \\
& \quad \leq\left\|(-A)^{-\delta}\left(e^{A(t-s)}-I\right)+\left(I-S_{\Delta t}\right)\right\|_{L(H)} \\
& \quad \leq C\left((t-s)^{\delta}+(\Delta t)^{\delta}\right),
\end{aligned}
$$

from which we get

$$
\int_{(k-1) \Delta t}^{k \Delta t}\left\|(-A)^{-\delta}\left(e^{A(t-s)}-S_{\Delta t}\right)\right\|_{L(H)}^{2} d s \leq C(\Delta t)^{1+2 \delta} .
$$

Thus from (73), after replacing (79) and (81) in (72), we obtain

$$
\begin{gathered}
E\left\|\sum_{l=0}^{k-1} \int_{l \Delta t}^{(l+1) \Delta t}\left(e^{A(t-s)}-S_{\Delta t}^{k-l}\right) B\left(X_{s}\right) d W_{s}^{N}\right\|_{H}^{2} \\
\quad \leq C\left((\Delta t)+(\Delta t)^{2 \gamma_{1}}+(\Delta t)^{1+2 \delta}\right),
\end{gathered}
$$

which gives

$$
E\left\|I I I_{2}\right\|_{H}^{2} \leq C(\Delta t)^{2 \gamma_{1}} \leq C(\Delta t)^{2 \tilde{\gamma}}, \quad \text { since } \tilde{\gamma}<\gamma_{1} \text {. }
$$

For the third term $\mathrm{III}_{3}$, we have

$$
\begin{aligned}
& E\left\|\sum_{l=0}^{k-1} \int_{l \Delta t}^{(l+1) \Delta t} S_{\Delta t}^{k-l}\left(B\left(X_{l \Delta t}\right)-B\left(Y_{l}^{N}\right)\right) d W_{s}^{N}\right\|_{H}^{2} \\
& \leq \sum_{l=0}^{k-1} \int_{l \Delta t}^{(l+1) \Delta t} E\left\|S_{\Delta t}^{k-l}\left(B\left(X_{l \Delta t}\right)-B\left(Y_{l}^{N}\right)\right)\right\|_{H S\left(U_{0}, H\right)} d s \\
& \leq \sum_{l=0}^{k-1} \int_{l \Delta t}^{(l+1) \Delta t} E\left\|S_{\Delta t}^{k-l}\right\|_{L(H)} \\
& \leq C \sum_{l=0}^{k-1} \int_{l \Delta t}^{(l+1) \Delta t} E\left\|\left(B\left(X_{l \Delta t}\right)-B\left(Y_{l}^{N}\right)\right)\right\|_{H S\left(U_{0}, H\right)} d s
\end{aligned}
$$

This implies that

$$
\begin{aligned}
& E\left\|I I_{3}\right\|_{H}^{2} \\
& \leq E\left\|\sum_{l=0}^{k-1} \int_{l \Delta t}^{(l+1) \Delta t} S_{\Delta t}^{k-l}\left(B\left(X_{l \Delta t}\right)-B\left(Y_{l}^{N}\right)\right) d W_{s}^{N}\right\|_{H}^{2} \\
& \leq C \sum_{l=0}^{k-1}(\Delta t) E\left\|e_{l}^{N}\right\|_{H^{*}}^{2}
\end{aligned}
$$

Finally for $\mathrm{III}_{4}$, the last term of (65), we should recall

$$
\begin{aligned}
& I I I_{4}=P_{N}\left(\sum_{l=0}^{k-1} \int_{l \Delta t}^{(l+1) \Delta t} S_{\Delta t}^{k-l}\right. \\
& \quad \times\left(B\left(X_{s}\right)-B\left(X_{l \Delta t}\right)\right) d W_{s}^{N} \\
& \quad-\sum_{l=0}^{k-1} \int_{l \Delta t}^{(l+1) \Delta t} S_{\Delta t}^{k-l} B^{\prime}\left(Y_{l}^{N}\right) \\
&\left.\quad \times\left(\int_{l \Delta t}^{s} B\left(Y_{l}^{N}\right) d W_{u}^{N}\right) d W_{s}^{N}\right) .
\end{aligned}
$$

Using the fact that

$$
\begin{aligned}
B\left(X_{s}\right)-B\left(X_{l \Delta t}\right) & \\
= & B^{\prime}\left(X_{l \Delta t}\right)\left(X_{s}-X_{l \Delta t}\right) \\
+ & \int_{0}^{1} B\left(X_{l \Delta t}+r\left(X_{s}-X_{l \Delta t}\right)\right)\left(X_{s}-X_{l \Delta t}, X_{s}-X_{l \Delta t}\right) \\
\quad & \times(1-r) d r
\end{aligned}
$$


for all $s \in[l \Delta t,(l+1) \Delta t], l \in\left\{0,1,2, \ldots, N^{2}-1\right\}, N \in \mathbb{N}$, and $r \in(0,1)$ and using the inequality

$$
\left(a_{1}+a_{2}+\cdots+a_{n}\right)^{2} \leq n\left(a_{1}^{2}+a_{2}^{2}+\cdots+a_{n}^{2}\right)
$$

for all $a_{i} \in \mathbb{R}, i=1, \ldots, N$, for $I I I_{4}$, we obtain

$$
\begin{aligned}
& E\left\|I I I_{4}\right\|_{H}^{2} \\
& \qquad 2 E \| \sum_{l=0}^{k-1} \int_{l \Delta t}^{(l+1) \Delta t} S_{\Delta t}^{k-l} B^{\prime}\left(X_{l \Delta t}\right) \\
& \times\left(X_{s}-X_{\Delta t}-\int_{l \Delta t}^{s} B\left(X_{l \Delta t}\right) d W_{u}^{N}\right) d W_{s}^{N} \|_{H}^{2} \\
& +2 E \| \int_{l \Delta t}^{(l+1) \Delta t} S_{\Delta t}^{k-l} \int_{0}^{1} B^{\prime \prime}\left(X_{l \Delta t}+r\left(X_{s}-X_{l \Delta t}\right)\right) \\
& \times\left(X_{s}-X_{l \Delta t}, X_{s}-X_{l \Delta t}\right) \\
& \times(1-r) d r d W_{s}^{N} \|_{H}^{2} .
\end{aligned}
$$

To estimate the first term of (89), we first approximate

$$
E\left\|X_{s}-X_{l \Delta t}-\int_{l \Delta t}^{s} B\left(X_{l \Delta t}\right) d W_{u}^{N}\right\|_{H}^{2}
$$

for all $l \Delta t, s \in[0, T]$, with $l \Delta t \leq s$ and all $N \in \mathbb{N}$. More precisely, with respect to (88), we have

$$
\begin{aligned}
E \| X_{s} & -X_{l \Delta t}-\int_{l \Delta t}^{s} B\left(X_{l \Delta t}\right) d W_{u}^{N} \|_{H}^{2} \\
\leq & 5 E\left\|\left(e^{A(s-l \Delta t)}-I\right) X_{l \Delta t}\right\|_{H}^{2} \\
& +5 E\left\|\int_{l \Delta t}^{s} e^{A(s-u)} F\left(X_{u}\right) d u\right\|_{H}^{2} \\
& +5 E\left\|\int_{l \Delta t}^{s} e^{A(s-u)} B\left(X_{u}\right) d\left(W_{u}-W_{u}^{N}\right)\right\|_{H}^{2} \\
& +5 E\left\|\int_{l \Delta t}^{s}\left(e^{A(s-u)}-I\right) B\left(X_{u}\right) d W_{u}^{N}\right\|_{H} \\
& +5 E\left\|\int_{l \Delta t}^{s}\left(B\left(X_{u}\right)-B\left(X_{s}\right)\right) d W_{u}^{N}\right\|_{H}^{2} .
\end{aligned}
$$

By using (71), we have

$$
\begin{aligned}
E \| X_{s} & -X_{l \Delta t}-\int_{l \Delta t}^{s} B\left(X_{l \Delta t}\right) d W_{u}^{N} \|_{H}^{2} \\
\leq & 5\left\|(-A)^{-\gamma}\left(e^{A(s-l \Delta t)}-I\right)\right\|_{L(H)}^{2} E\left\|(-A)^{\gamma} X_{l \Delta t}\right\|_{H}^{2} \\
& +5(s-l \Delta t)\left(\int_{l \Delta t}^{s} E\left\|e^{A(s-u)} F\left(X_{u}\right)\right\|_{H}^{2} d u\right) \\
& +C\left(\sup _{j>N+1} \eta_{j}\right)^{2 \alpha} \\
& +5\left(\int_{l \Delta t}^{s} E\left\|\left(e^{A(s-u)}-I\right) B\left(X_{u}\right)\right\|_{H S\left(U_{0}, H\right)}^{2} d u\right) \\
& +5\left(\int_{l \Delta t}^{s} E\left\|B\left(X_{u}\right)-B\left(X_{l \Delta t}\right)\right\|_{H S\left(U_{0}, H\right)}^{2} d u\right) .
\end{aligned}
$$

This implies that

$$
\begin{aligned}
E \| X_{s} & -X_{l \Delta t}-\int_{l \Delta t}^{s} B\left(X_{l \Delta t}\right) d W_{u}^{N} \|_{H}^{2} \\
\leq & 5 R(s-l \Delta t)^{2 \gamma}+5(s-l \Delta t) \\
& \times\left(\int_{l \Delta t}^{s} E\left\|F\left(X_{u}\right)\right\|_{H}^{2} d u\right)+C\left(\sup _{j>N+1} \eta_{j}\right)^{2 \alpha} \\
& +5\left(\int_{l \Delta t}^{s}\left\|(-A)^{-\delta}\left(e^{A(s-u)}-I\right)\right\|_{L(H)}^{2}\right. \\
& \left.\times E\left\|(-A)^{\delta} B\left(X_{u}\right)\right\|_{H S\left(U_{0}, H\right)}^{2} d u\right) \\
& +5 R^{2}\left(\int_{l \Delta t}^{s} E\left\|X_{u}-X_{l \Delta t}\right\|_{H}^{2} d u\right) \\
\leq & 5 R(s-l \Delta t)^{2 \gamma}+5 R(s-l \Delta t)^{2}+C\left(\sup _{j>N+1} \eta_{j}\right)^{2 \alpha} \\
& +5\left(\int_{l \Delta t}^{s}\left\|(-A)^{-\delta}\left(e^{A(s-u)}-I\right)\right\|_{L(H)}^{2}\right. \\
& \times 5 R^{2}\left(\int_{l \Delta t}^{s}\left\|(-A)^{-\beta}\right\|_{L(H)} E\left\|X_{u}-X_{s}\right\|_{V_{\beta}}^{2} d u\right) \\
& +5\left(\int_{l \Delta t}^{s}(s-u)^{2 \delta} E\left\|(-A)^{\delta} B\left(X_{u}\right)\right\|_{H s\left(U_{0}, H\right)}^{2} B\left(X_{u}\right) \|_{H s\left(U_{0}, H\right)}^{2} d u\right) \\
& +5 R^{2}\left(\int_{l \Delta t}^{s} E\left\|X_{u}-X_{l \Delta t}\right\|_{H}^{2} d u\right) \\
\leq & 5 R(s-l \Delta t)^{2 \gamma} \\
& +5 R(s-l \Delta t)^{2}+C\left(\sup _{j>N+1} \eta_{j}\right)^{2 \alpha}
\end{aligned}
$$


for all $l \Delta t, s \in[0, T]$, with $l \Delta t \leq s$, and all $k \in \mathbb{N}$. Therefore, we obtain

$$
\begin{aligned}
E \| X_{s} & -X_{l \Delta t}-\int_{l \Delta t}^{s} B\left(X_{l \Delta t}\right) d W_{u}^{N} \|_{H}^{2} \\
\leq & 5 R(s-l \Delta t)^{2 \gamma}+5 R(s-l \Delta t)^{2} \\
& +C\left(\sup _{j>N+1} \eta_{j}\right)^{2 \alpha}+5 R\left(\int_{l \Delta t}^{s}(s-u)^{2 \delta} d u\right) \\
& +5 R^{4}\left(\int_{l \Delta t}^{s} E\left\|X_{u}-X_{l \Delta t}\right\|_{V_{\beta}}^{2} d u\right) \\
\leq & 10 R^{3}(s-l \Delta t)^{2 \gamma}+C\left(\sup _{j>N+1} \eta_{j}\right)^{2 \alpha} \\
& +5 R(s-l \Delta t)^{1+2 \delta} \\
& +5 R^{4}\left(\int_{l \Delta t}^{s} E\left\|X_{u}-X_{l \Delta t}\right\|_{V_{\beta}}^{2} d u\right) \\
\leq & 15 R^{3}(s-l \Delta t)^{2 \gamma}+C\left(\sup _{j>N+1} \eta_{j}\right)^{2 \alpha} \\
& +5 R^{4}\left(\int_{l \Delta t}^{s}\left(E\left\|X_{u}-X_{l \Delta t}\right\|_{V_{\beta}}^{4}\right)^{1 / 2} d u\right) \\
\leq & 15 R^{3}(s-l \Delta t)^{2 \gamma}+C\left(\sup _{j>N+1} \eta_{j}\right)^{2 \alpha} \\
& +5 R^{4}\left(\int_{l \Delta t}^{s} R(u-l \Delta t)^{\min (4(\gamma-\beta), 2)}\right)^{1 / 2} d u
\end{aligned}
$$

and hence

$$
\begin{gathered}
E\left\|X_{s}-X_{l \Delta t}-\int_{l \Delta t}^{s} B\left(X_{l \Delta t}\right) d W_{u}^{N}\right\|_{H}^{2} \\
\leq 15 R^{3}\left((s-l \Delta t)^{2 \gamma}+C\left(\sup _{j>N+1} \eta_{j}\right)^{2 \alpha}\right. \\
\left.+5 R^{5}\left(\int_{l \Delta t}^{s}(u-l \Delta t)^{\min (4(\gamma-\beta), 2)}\right) d u\right) \\
\leq 15 R^{3}\left((s-l \Delta t)^{2 \gamma}+C\left(\sup _{j>N+1} \eta_{j}\right)^{2 \alpha}\right. \\
\left.\quad+5 R^{5}(s-l \Delta t)^{1+\min (4(\gamma-\beta), 2)}\right) \\
\leq C\left((s-l \Delta t)^{\min (4(\gamma-\beta), 2 \gamma)}+\left(\sup _{j>N+1} \eta_{j}\right)^{2 \alpha}\right)
\end{gathered}
$$

for all $l \Delta t, s \in[0, T]$, with $l \Delta t \leq s$.
Therefore,

$$
\begin{gathered}
E \| \sum_{l=0}^{k-1} \int_{l \Delta t}^{l+1 \Delta t} S_{\Delta t}^{k-l} B^{\prime}\left(X_{l \Delta t}\right) \\
\quad \times\left(X_{s}-X_{l \Delta t}-\int_{l \Delta t}^{s} B\left(X_{l \Delta t}\right) d W_{u}^{N}\right) d W_{s}^{N} \|_{H}^{2} \\
\leq \sum_{l=0}^{k-1} \int_{l \Delta t}^{l+1 \Delta t} E \\
\times \| B^{\prime}\left(X_{l \Delta t}\right) \\
\quad \times\left(X_{s}-X_{l \Delta t}\right. \\
\left.\quad-\int_{l \Delta t}^{s} B\left(X_{l \Delta t}\right) d W_{u}^{N}\right) d W_{s}^{N} \|_{H S\left(U_{0}, H\right)}^{2} d s \\
\quad \times \sum_{l=0}^{k-1} \int_{l \Delta t}^{(l+1) \Delta t} E \\
\left.\quad\left\|X_{s}-X_{l \Delta t}-\int_{l \Delta t}^{s} B\left(X_{l \Delta t}\right) d W_{u}^{N}\right\|_{H}^{2} d s\right)
\end{gathered}
$$

and hence from (95), we get

$E \| \sum_{l=0}^{k-1} \int_{l \Delta t}^{(l+1) \Delta t} S_{\Delta t}^{k-l} B^{\prime}\left(X_{l \Delta t}\right)$

$$
\begin{gathered}
\times\left(X_{s}-X_{l \Delta t}-\int_{l \Delta t}^{s} B\left(X_{l \Delta t}\right) d W_{u}^{N}\right) d W_{s}^{N} \|_{H}^{2} \\
\leq C\left(\sum _ { l = 0 } ^ { k - 1 } \int _ { l \Delta t } ^ { ( l + 1 ) \Delta t } \left((s-l \Delta t)^{\min (4(\gamma-\beta), 2 \gamma)}\right.\right. \\
\left.\left.+\left(\sup _{j>N+1} \eta_{j}\right)^{2 \alpha}\right) d s\right) \\
\leq C\left(\sum_{l=0}^{k-1} \int_{l \Delta t}^{(l+1) \Delta t}(s-l \Delta t)^{\min (4(\gamma-\beta), 2 \gamma)} d s\right. \\
\left.+\left(\sup _{j>N+1} \eta_{j}\right)^{2 \alpha}\right) \\
\leq C\left(N^{2}(\Delta t)^{(1+\min (4(\gamma-\beta), 2 \gamma))}+\left(\sup _{j>N+1} \eta_{j}\right)^{2 \alpha}\right) \\
\leq C\left((\Delta t)^{\min (4(\gamma-\beta), 2 \gamma)}+\left(\sup _{j>N+1} \eta_{j}\right)^{2 \alpha}\right) .
\end{gathered}
$$


And for the second term of (89), we have

$$
\begin{aligned}
& E \| \sum_{l=0}^{k-1} \int_{l \Delta t}^{(l+1) \Delta t} S_{\Delta t}^{k-l} \int_{0}^{1} B^{\prime \prime}\left(X_{l \Delta t}+r\left(X_{s}-X_{l \Delta t}\right)\right) \\
& \times\left(X_{s}-X_{l \Delta t}, X_{s}-X_{l \Delta t}\right) \\
& \times(1-r) d r d W_{s}^{N} \|_{H}^{2} \\
& \leq \sum_{l=0}^{k-1} \int_{l \Delta t}^{(l+1) \Delta t} \int_{0}^{1} E \| B^{\prime \prime}\left(X_{l \Delta t}+r\left(X_{s}-X_{l \Delta t}\right)\right) \\
& \times\left(X_{s}-X_{l \Delta t},\right. \\
& \left.X_{s}-X_{l \Delta t}\right) \|_{H S\left(U_{0}, H\right)}^{2} d r d s \\
& \leq \sum_{l=0}^{k-1} \int_{l \Delta t}^{(l+1) \Delta t} E\left[\left(R\left\|X_{s}-X_{l \Delta t}\right\|_{V_{\beta}}^{2}\right)^{2}\right] d s \\
& \leq R^{2} \sum_{l=0}^{k-1} \int_{l \Delta t}^{(l+1) \Delta t} E\left\|X_{s}-X_{l \Delta t}\right\|_{V_{\beta}}^{4} d s \\
& \leq R^{2}\left(\sum_{l=0}^{k-1} \int_{l \Delta t}^{(l+1) \Delta t} R(S-l \Delta t)^{\min (4(\gamma-\beta), 2)} d s\right. \\
& \left.\leq R^{3} \sum_{l=0}^{k-1}(\Delta t)^{1+\min (4(\gamma-\beta), 2)}\right) \\
& \leq C(\Delta t)^{\min (4(\gamma-\beta), 2)} .
\end{aligned}
$$

Therefore, from (71), (83), (85), (89), (97), and (98), we obtain

$$
\begin{aligned}
& E\|I I I\|_{H}^{2} \\
& \leq C\left(\left(\sup _{j>N+1} \mu_{j}\right)^{2 \alpha}+(\Delta t)^{2 \widehat{\gamma}}+\sum_{l=0}^{k-1}(\Delta t) E\left\|\hat{e}_{l}^{N}\right\|_{H}^{2}\right) \\
& +(\Delta t)^{\min (4(\gamma-\beta), 2 \gamma)}+\left(\sup _{j>N+1} \mu_{j}\right)^{2 \alpha} \\
& +(\Delta t)^{\min (4(\gamma-\beta), 2 \gamma)} \\
& \leq \quad\left(\left(\sup _{j>N+1} \mu_{j}\right)^{2 \alpha}+(\Delta t)^{\min (4(\gamma-\beta), 2 \gamma)}\right. \\
& \left.\quad+\sum_{l=0}^{k-1}(\Delta t) E\left\|\hat{e}_{l}^{N}\right\|_{H}^{2}\right) .
\end{aligned}
$$

Hence from (50), (63), and (100), we obtain

$$
\begin{aligned}
& \left(E\left\|\widehat{e}_{k}^{N}\right\|_{H}^{2}\right)^{1 / 2} \\
& \quad \leq C\left(\frac{\left(E\left\|X_{0}\right\|_{H}^{2}\right)^{1 / 2}}{k}+\left(E\left\|\hat{e}_{0}^{N}\right\|_{H}^{2}\right)^{1 / 2}+(\Delta t)^{\gamma}\right.
\end{aligned}
$$

$$
\begin{aligned}
& +(\Delta t)^{\min \{\gamma, 1 / 2\}}+\sum_{l=0}^{k-1}(\Delta t)\left(E\left\|e_{k}^{N}\right\|_{H}^{2}\right)^{1 / 2} \\
& +\left(\sup _{j>N+1} \mu_{j}\right)^{\alpha} \\
& \left.+(\Delta t)^{\min (2(\gamma-\beta), \gamma)}+\sum_{l=0}^{k-1}(\Delta t)\left(E\left\|\hat{e}_{k}^{N}\right\|_{H}^{2}\right)^{1 / 2}\right) .
\end{aligned}
$$

Now we take an integer $q \geq 1$ and use the Holder inequality for the two summations in the last estimation to get

$$
\begin{gathered}
\sum_{l=0}^{k-1}(\Delta t)\left(E\left\|\hat{e}_{l}^{N}\right\|_{H}^{2}\right)^{1 / 2} \\
\leq\left(\sum_{l=0}^{k-1} \Delta t\right)^{(2 q-1) / 2 q} \\
\times\left(\sum_{l=0}^{k-1}(\Delta t)\left(E\left\|\hat{e}_{l}^{N}\right\|_{H}^{2}\right)^{2 q / 2}\right)^{1 / 2 q} \\
\leq C\left(\sum_{l=0}^{k-1}(\Delta t)\left(E\left\|\hat{e}_{l}^{N}\right\|_{H}^{2}\right)^{q}\right)^{1 / 2 q} .
\end{gathered}
$$

Therefore, with using (97) and (98), we obtain

$$
\begin{gathered}
\left(E\left\|\widehat{e}_{k}^{N}\right\|_{H}^{2}\right)^{q} \\
\leq R\left(\left(\frac{\left(E\left\|X_{0}\right\|_{H}^{2}\right)^{1 / 2}}{k}+\left(E\left\|\hat{e}_{0}^{N}\right\|_{H}^{2}\right)^{1 / 2}\right.\right. \\
\left.+(\Delta t)^{\min (2(\gamma-\beta), \gamma)}+\left(\sup _{j>N+1} \mu_{j}\right)^{\alpha}\right)^{2 q} \\
+\sum_{l=0}^{k-1}(\Delta t)\left(E\left\|\hat{e}_{k}^{N}\right\|_{H}^{2}\right)^{q} \\
\left.+\sum_{l=0}^{k-1}(\Delta t)\left(E\left\|e_{k}^{N}\right\|_{H}^{2}\right)^{q}\right)
\end{gathered}
$$

We then have

$$
\begin{aligned}
& \left(E\left\|\widehat{e}_{k}^{N}\right\|_{H}^{2}\right)^{q} \\
& \quad \leq C\left(\left(\frac{\left(E\left\|X_{0}\right\|_{H}^{2}\right)^{1 / 2}}{k}+\left(E\left\|\hat{e}_{0}^{N}\right\|_{H}^{2}\right)^{1 / 2}\right.\right.
\end{aligned}
$$




$$
\begin{aligned}
& \left.+(\Delta t)^{\min (2(\gamma-\beta), \gamma)}+\left(\sup _{j>N+1} \mu_{j}\right)^{\alpha}\right)^{2 q} \\
& \left.+(1+\Delta t) \sum_{l=0}^{k-1}(\Delta t)\left(E\left\|\hat{e}_{k}^{N}\right\|_{H}^{2}\right)^{q}\right) .
\end{aligned}
$$

Hence, we conclude from (41) that

$$
\begin{gathered}
\left(E\left\|\hat{e}_{k}^{N}\right\|_{H}^{2}\right)^{1 / 2} \leq C\left(\frac{\left(E\left\|X_{0}\right\|_{H}^{2}\right)^{1 / 2}}{k}+\left(\sup _{j>N+1} \mu_{j}\right)^{\alpha}\right. \\
\left.+(\Delta t)^{\min (2(\gamma-\beta), \gamma)}\right) .
\end{gathered}
$$

Finally, with respect to (47) and (105), we obtain

$$
\begin{aligned}
& \left(E\left\|e_{k}^{N}\right\|_{H}^{2}\right)^{1 / 2} \\
& \leq C\left(\left(\inf _{j>N+1} \lambda_{j}\right)^{-\gamma}+\frac{\left(E\left\|X_{0}\right\|_{H}^{2}\right)^{1 / 2}}{k}+\left(\sup _{j>N+1} \mu_{j}\right)^{\alpha}\right. \\
& \left.\quad+(\Delta t)^{\min (2(\gamma-\beta), \gamma)}\right)
\end{aligned}
$$

which completes the proof of the theorem.

\section{Simulation Results}

In this section we consider SPDE (1) and solve it by numerical scheme (22). More formally, let $k=1 / 100$ and $\xi:[0,1] \rightarrow$ $\mathbb{R}$ be given by $\xi(x)=0$ for all $x \in[0,1]$ and suppose that $f, b:(0,1) \times \mathbb{R} \rightarrow \mathbb{R}$ are given by $f(x, y)=1-y$ and $b(x, y)=(1-y) /\left(1+y^{2}\right)$ for all $x \in(0,1), y \in \mathbb{R}$. The SPDE (1) reduces to

$$
\begin{aligned}
d X_{t}(x)= & {\left[\frac{1}{100} \frac{\partial^{2}}{\partial x^{2}} X_{t}(x)+1-X_{t}(x)\right] d t } \\
& +\frac{1-X_{t}(x)}{1+X_{t}^{2}(x)} d W_{t}(x)
\end{aligned}
$$

with $X_{t}(0)=X_{t}(1)=0$ and $X_{0}=0$ for $x \in(0,1)$ and $t \in[0,1]$. We also assume that the SPDE (107) should be solved with a precision of, say, two decimals, that is, with the precision $\varepsilon=0.01$ in (3). To confirm numerically our theoretical founding in Theorem 7 , we recall that for SPDE (107) there should exist some real number $C_{r} \in(0, \infty)$ such that

$$
\left(E\left\|X_{T}-Y_{N^{2}}^{N}\right\|_{H}^{2}\right)^{1 / 2} \leqslant C_{r} N^{r-3 / 2}
$$

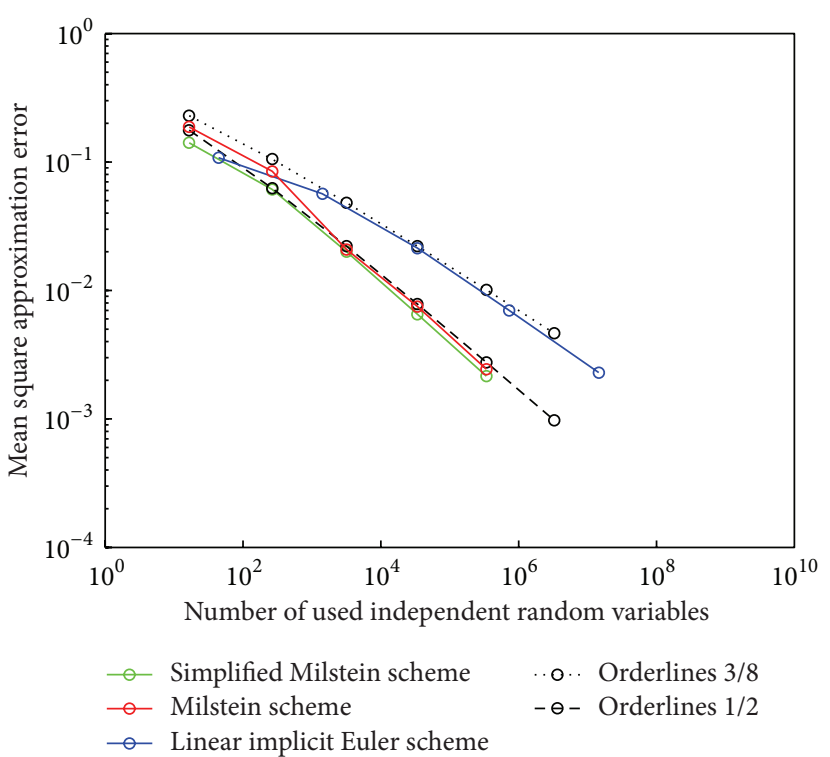

Figure 1: Approximation error in the sense of (22) of the linear implicit Euler and simplified Milstein and Milstein type schemes against the precise number of independent standard normal random variables needed to compute the corresponding approximation for $N \in\{2,4,8,16,32,64\}$.

holds for each small $r \in(0,3 / 4)$. The overall convergence order of the linear implicit Euler method (4) is 3/8- (see [16]), while the overall convergence of the simplified Milstein scheme (22) and Milstein scheme (7) is 1/2. In Figure 1 the approximation error in the sense of (6) of the linear implicit Euler approximation $\bar{Y}_{N^{3}}^{N}$, obtained by (4), of the approximation $\widetilde{Y}_{N^{2}}^{N}$, obtained by Milstein scheme (7), and of the approximation $Y_{N^{2}}^{N}$, obtained by simplified Milstein scheme (22), is plotted against the precise number of independent standard normal random variables that is needed to compute the corresponding approximation for $N \in\{2,4,8, \ldots, 128\}$ on a log-log scale. Figure 1 confirms the order of convergence of our scheme and compares with the other two schemes. Besides, the simplified Milstein scheme (22) and the Milstein scheme (7) produce nearly the same approximation errors. Numerical results also show that the linear simplified Milstein scheme (21) and the Milstein type scheme (7) are much computationally effective than the linear implicit Euler scheme (4). To simulate one path $\bar{Y}_{32^{3}}^{32}$, one needs to generate $32^{4}=1048576$ independent normal random variables, but this amount for simulation of $\widetilde{Y}_{32^{2}}^{32}$ and $Y_{32^{2}}^{32}$ reduces to $32^{3}=$ 32768. From the numerical results reported in Table 1 and Figure 1, we conclude that the simplified Milstein scheme is more effective than implicit Euler method and slightly better than Milstein scheme.

\section{Conclusions}

A simplified Milstein scheme for solving stochastic partial differential equations of the form (1) with multiplicative trace class noise was theoretically and numerically investigated. 
This scheme has advantages to some other methods such as linear implicit Euler and Milstein schemes. We have shown the $L^{2}$ convergence of this method under the stated conditions and then we have illustrated the effectiveness of the simplified Milstein scheme numerically.

\section{Conflict of Interests}

The authors declare that there is no conflict of interests regarding the publication of this paper.

\section{References}

[1] G. da Prato and J. Zabczyk, Stochastic Equations in Infinite Dimensions, vol. 44, Cambridge University Press, Cambridge, UK, 1992.

[2] Y. S. Mishura and G. M. Shevchenko, "Approximation schemes for stochastic differential equations in a Hilbert space," Teoriya Veroyatnostei i ee Primeneniya, vol. 51, no. 3, pp. 476-495, 2006.

[3] J. Printems, "On the discretization in time of parabolic stochastic partial differential equations," Mathematical Modelling and Numerical Analysis, vol. 35, no. 6, pp. 1055-1078, 2001.

[4] P. E. Kloeden and E. Platen, Numerical Solutions of Stochastic Differential Equations, Springer, Berlin, Germany, 1992.

[5] T. Shardlow, "Numerical methods for stochastic parabolic PDEs," Numerical Functional Analysis and Optimization, vol. 20, no. 1-2, pp. 121-145, 1999.

[6] I. Gyöngy and N. Krylov, "An accelerated splitting-up method for parabolic equations," SIAM Journal on Mathematical Analysis, vol. 37, no. 4, pp. 1070-1097, 2005.

[7] A. M. Davie and J. G. Gaines, "Convergence of numerical schemes for the solution of parabolic stochastic partial differential equations," Mathematics of Computation, vol. 70, no. 233, pp. 121-134, 2001.

[8] E. Hausenblas, "Numerical analysis of semilinear stochastic evolution equations in Banach spaces," Journal of Computational and Applied Mathematics, vol. 147, no. 2, pp. 485-516, 2002.

[9] A. Lang, P. Chow, and J. Potthoff, "Almost sure convergence for a semidiscrete Milstein scheme for SPDEs of Zakai type," Stochastics, vol. 82, no. 1-3, pp. 315-326, 2010.

[10] A. Jentzen, P. Kloeden, and G. Winkel, "Efficient simulation of nonlinear parabolic SPDEs with additive noise," The Annals of Applied Probability, vol. 21, no. 3, pp. 908-950, 2011.

[11] P. E. Kloeden and S. Shott, "Linear-implicit strong schemes for Itô-Galerkin approximations of stochastic PDEs," Journal of Applied Mathematics and Stochastic Analysis, vol. 14, no. 1, pp. 47-53, 2001.

[12] Y. Yan, "Galerkin finite element methods for stochastic parabolic partial differential equations," SIAM Journal on Numerical Analysis, vol. 43, no. 4, pp. 1363-1384, 2005.

[13] J. B. Walsh, "On numerical solutions of the stochastic wave equation," Illinois Journal of Mathematics, vol. 50, no. 1-4, pp. 991-1018, 2006.

[14] R. Kruse, "Consistency and stability of a Milstein-Galerkin finite element scheme for semilinear SPDE," http://arxiv.org/ abs/1307.4120.

[15] C. Prévôt and M. Röckner, A Concise Course on Stochastic Partial Differential Equations, vol. 1905 of Lecture Notes in Mathematics, Springer, Berlin, Germany, 2007.
[16] A. Jentzen and M. Rockner, "A Milstein scheme for SPDEs," submitted, http://arxiv.org/abs/1001.2751.

[17] A. Jentzen and M. Röckner, "Regularity analysis for stochastic partial differential equations with nonlinear multiplicative trace class noise," Journal of Differential Equations, vol. 252, no. 1, pp. 114-136, 2012. 


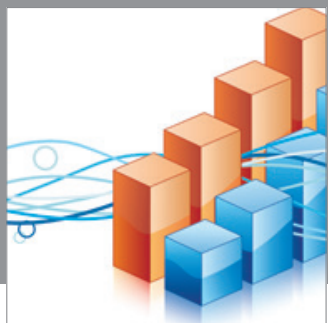

Advances in

Operations Research

mansans

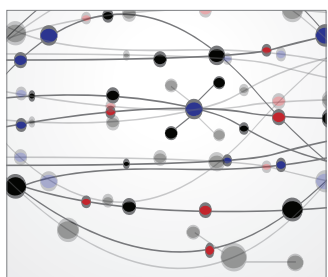

The Scientific World Journal
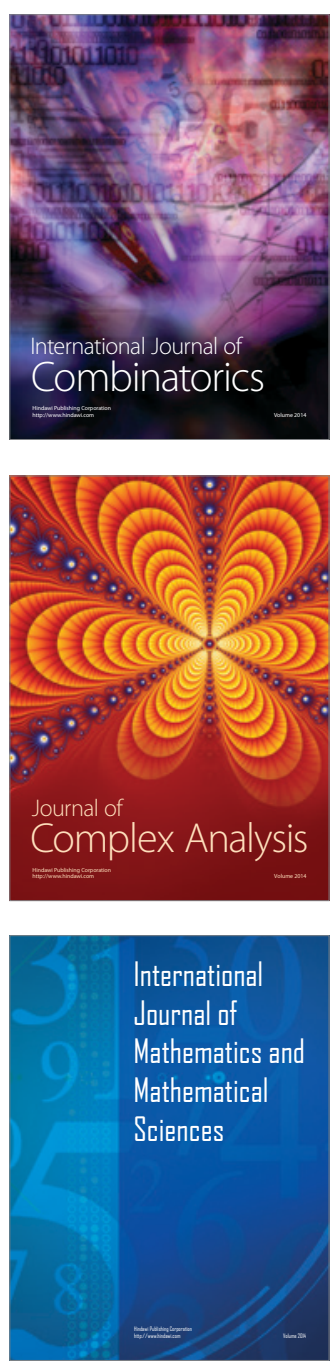
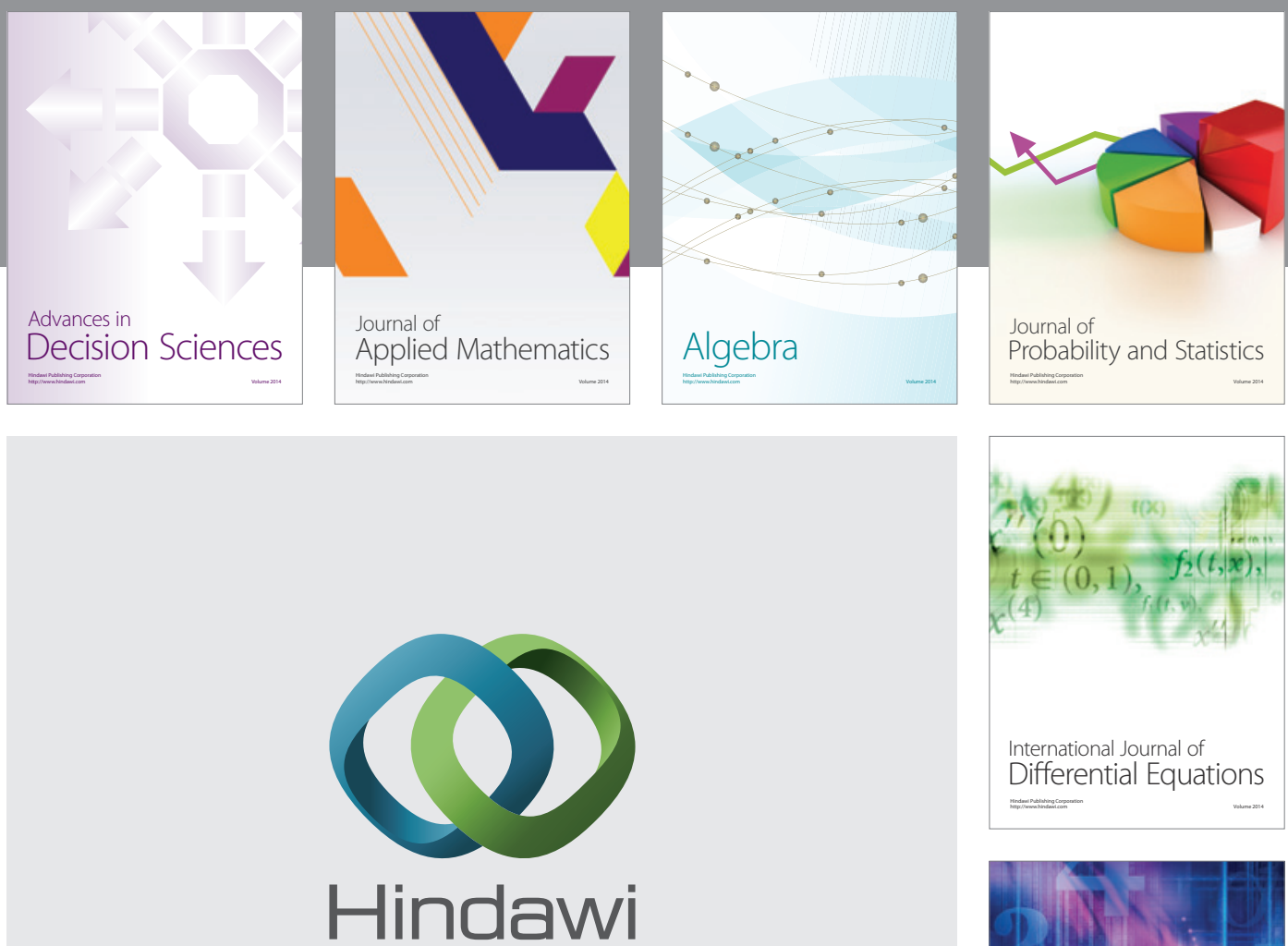

Submit your manuscripts at http://www.hindawi.com
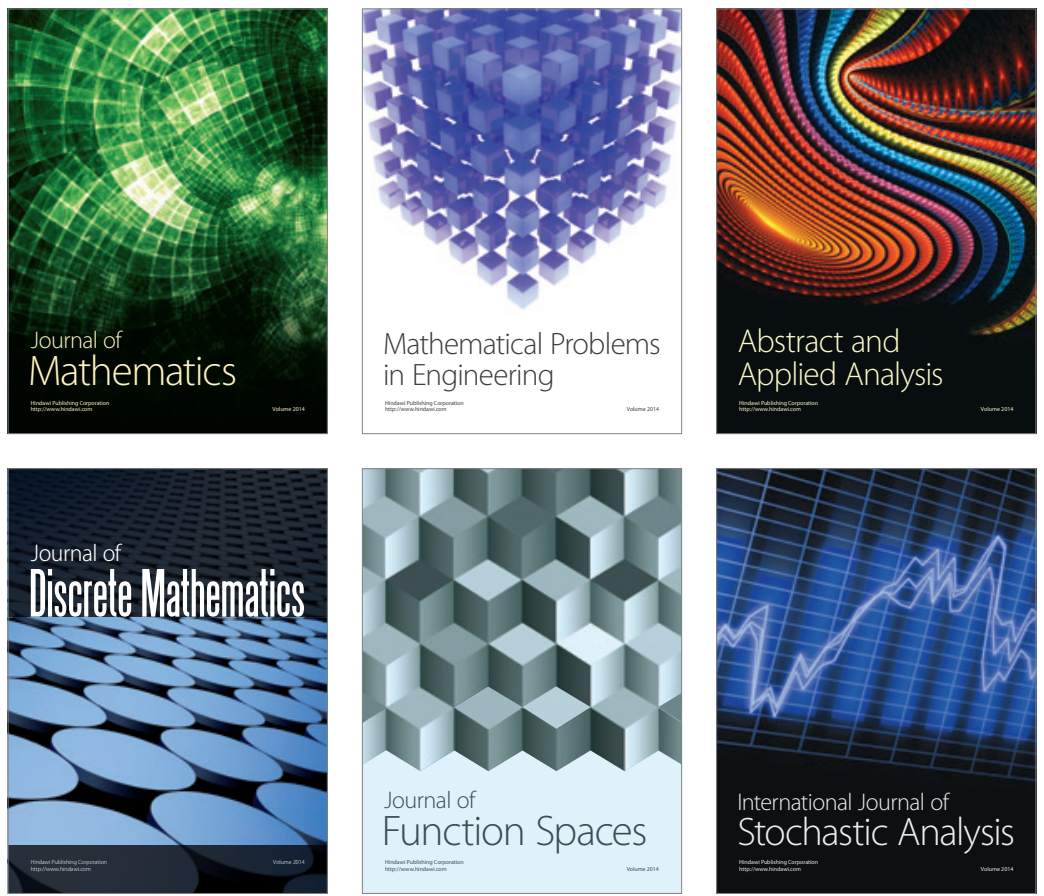

Journal of

Function Spaces

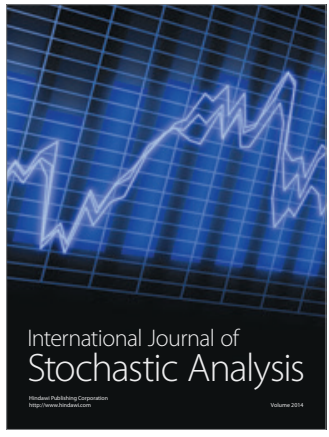

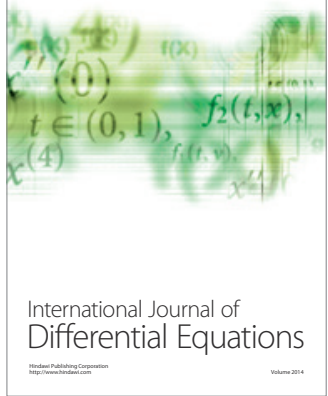
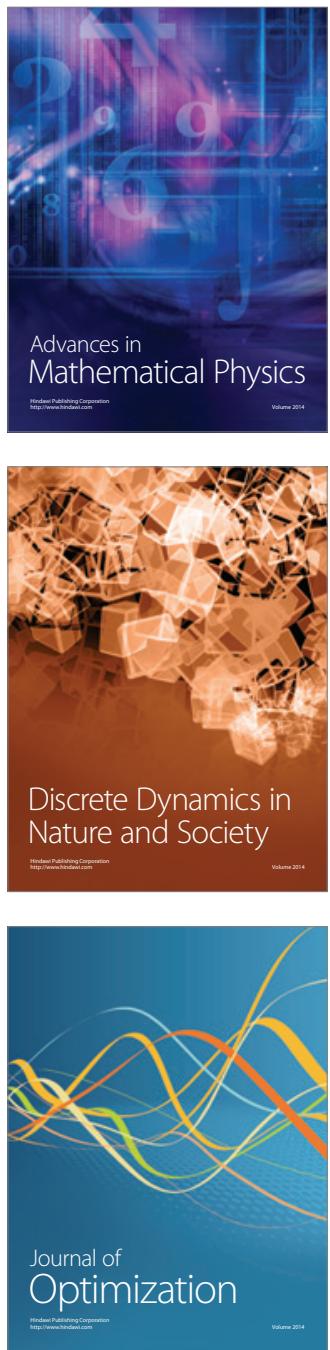